\title{
Autophagy impairment as a key feature for acetaminophen-induced ototoxicity
}

\author{
Tong Zhao', Tihua Zheng ${ }^{1}$, Huining $\mathrm{Yu}^{1}$, Bo Hua $\mathrm{Hu}^{2}$, Bing $\mathrm{Hu}^{3}$, Peng $\mathrm{Ma}^{4}$, Ying Yang ${ }^{1}$, Naidi Yang ${ }^{5}$, Juan $\mathrm{Hu}^{6}$, \\ Tongtao Cao ${ }^{1}$, Gang Chen ${ }^{7}$, Bin Yan ${ }^{1}$, Melina Peshoff ${ }^{8}$, Maria Hatzoglou' ${ }^{9}$, Ruishuang Geng ${ }^{1}$, Bo Li id] ${ }^{1}$ and \\ Qing Yin Zheng ${ }^{8}$
}

\begin{abstract}
Macroautophagy/autophagy is a highly conserved self-digestion pathway that plays an important role in cytoprotection under stress conditions. Autophagy is involved in hepatotoxicity induced by acetaminophen (APAP) in experimental animals and in humans. APAP also causes ototoxicity. However, the role of autophagy in APAP-induced auditory hair cell damage is unclear. In the present study, we investigated autophagy mechanisms during APAPinduced cell death in a mouse auditory cell line (HEI-OC1) and mouse cochlear explant culture. We found that the expression of LC3-II protein and autophagic structures was increased in APAP-treated HEI-OC1 cells; however, the degradation of SQSTM1/p62 protein, the yellow puncta of mRFP-GFP-LC3 fluorescence, and the activity of lysosomal enzymes decreased in APAP-treated HEl-OC1 cells. The degradation of p62 protein and the expression of lysosomal enzymes also decreased in APAP-treated mouse cochlear explants. These data indicate that APAP treatment compromises autophagic degradation and causes lysosomal dysfunction. We suggest that lysosomal dysfunction may be directly responsible for APAP-induced autophagy impairment. Treatment with antioxidant N-acetylcysteine (NAC) partially alleviated APAP-induced autophagy impairment and apoptotic cell death, suggesting the involvement of oxidative stress in APAP-induced autophagy impairment. Inhibition of autophagy by knocking down of Atg5 and Atg7 aggravated APAP-induced ER and oxidative stress and increased apoptotic cell death. This study provides a better understanding of the mechanism responsible for APAP ototoxicity, which is important for future exploration of treatment strategies for the prevention of hearing loss caused by ototoxic medications.
\end{abstract}

\section{Introduction}

Acetaminophen ( $N$-acetyl-p-aminophenol [APAP], also known as paracetamol) is widely used as a pain reliever and fever reducer. APAP is effective and safe at the therapeutic dosage. However, APAP overdose can cause severe hepatotoxicity in experimental animals and humans ${ }^{1,2}$. APAP-induced hepatotoxicity has been recognized for $>50$ years and its underlying mechanism is

\footnotetext{
Correspondence: Ruishuang Geng (gengruishuang@hotmail.com) or Bo Li (liboyjt@163.com)

${ }^{1}$ Hearing and Speech Rehabilitation Institute, College of Special Education, Binzhou Medical University, Yantai, China

${ }^{2}$ Center for Hearing and Deafness, University at Buffalo, Buffalo, NY, USA

Full list of author information is available at the end of the article

These authors contributed equally: Tong Zhao, Tihua Zheng, Huining Yu

Edited by F. Strappazzon
}

fairly well known. APAP is metabolized by a cytochrome P450 2E1 isozyme (CYP2E1) to a reactive intermediate, $\mathrm{N}$-acetyl-p-benzoquinone imine (NAPQI). NAPQI causes the depletion of glutathione and the formation of APAP-cysteine adducts ${ }^{3,4}$. The APAP adducts lead to mitochondrial dysfunction and oxidative stress, which is a critical step in triggering hepatocyte necrosis and liver injury ${ }^{5,6}$. A previous study indicated that APAP adducts are removed through selective autophagy in primary mouse hepatocytes. The pharmacological induction of autophagy may be a novel and promising approach for treating APAP-induced liver injury ${ }^{7}$.

Autophagy, meaning "self-eating" in Greek, is an important cellular degradation process that occurs in response to physiological or chemical stresses ${ }^{8,9}$.

\section{(c) The Author(s) 2021}

(c) (i) Open Access This article is licensed under a Creative Commons Attribution 4.0 International License, which permits use, sharing, adaptation, distribution and reproduction in any medium or format, as long as you give appropriate credit to the original author(s) and the source, provide a link to the Creative Commons license, and indicate if changes were made. The images or other third party material in this article are included in the article's Creative Commons license, unless indicated otherwise in a credit line to the material. If material is not included in the article's Creative Commons license and your intended use is not permitted by statutory regulation or exceeds the permitted use, you will need to obtain permission directly from the copyright holder. To view a copy of this license, visit http://creativecommons.org/licenses/by/4.0/. 
Autophagy activation generates autophagosomes, which subsequently fuse with lysosomes to form autolysosomes. The lysosomal hydrolases degrade intra-autophagosomal contents to produce amino acids, which are used for the synthesis of macromolecules and for the production of energy $^{10}$. Microtubule-associated protein $1 \mathrm{~A} / 1 \mathrm{~B}$ light chain 3 (LC3) is ubiquitous in mammalian cells and tissues. LC3-II (a cleaved and phospholipid-conjugated form of LC3) and SQSTM1/p62 (sequestosome 1) are biomarkers for active autophagosomes. LC3-II is present in the membranes of autophagosomes and is degraded (along with p62, a cargo of autophagosomes) by lysosomal acidic enzymes after fusion of the lysosome and autophagosome. Incomplete maturation and a decrease in lysosome activity lead to decreased autolysosome formation and increased LC3-II and p62 accumulation in autophagosome membranes. Recent reports have shown that autophagy has protective effects, but impaired autophagy may induce cell death ${ }^{7,11-14}$.

Hair cells (HCs) in the cochlea play a critical role in converting mechanical signals into neural signals for hearing ${ }^{15}$ and are vulnerable to multiple types of damage, including acoustic trauma, ototoxicity, inflammation, and aging $^{16-20}$. Autophagy plays an important role during the development of the inner ear and is essential for the survival of HCs under various ototoxic conditions ${ }^{13,16,21-23}$. In contrast to well-documented hepatotoxicity, APAPinduced ototoxicity has only recently been recognized in studies $^{24,25}$. Clinical evidence indicates that the abuse of analgesics containing APAP/hydrocodone or APAP/ codeine can cause hearing loss ${ }^{26,27}$. However, the mechanisms underlying the ototoxic effects of these drugs have yet to be fully characterized. Yorgason et al. reported that APAP, but not hydrocodone, was the primary ototoxic agent in neonatal mouse cochlear cultures and in a mouse HC-like auditory cell line (HEI-OC1), though the mechanism behind its toxic effects remains unknown ${ }^{28}$. Kalinec et al. investigated the mechanisms of APAP and NAPQI cytotoxicity in the HEI-OC1 cell line ${ }^{29}$. Their study suggested that APAP causes HEI-OC1 cell death by inducing oxidative stress, and the toxic effects of both APAP and NAPQI in HEI-OC1 cells involve the induction of endoplasmic reticulum (ER) stress signaling through controlling the protein kinase RNA-like ER kinases (PERK).

Furthermore, it has been suggested that an APAPinduced increase in autophagy could exert a cytoprotective effect against APAP-induced apoptosis in auditory cells $^{30}$. Previous results have shown that Beclin-1 and LC3-II proteins increase following APAP exposure. However, the expression levels of the autophagy protein SQSTM1/p62, a cargo receptor involved in the selective degradation of ubiquitinated aggregates/organelles by autophagy, has not yet been determined in APAP-treated auditory cells. Thus, the autophagy mechanisms responsible for APAP ototoxicity in auditory cells remain unclear.

Therefore, we investigated whether the autophagy pathway plays a cytoprotective or cytotoxic role in APAP ototoxicity using two in vitro models: the HEI-OC1 cell line and mouse cochlear explants. We also examined the relationships between autophagy, oxidative stress, ER stress, and apoptosis signaling crosstalk in auditory cells. Our study offers novel insights into the APAP-induced ototoxicity mechanism and has the potential to lead to future treatment strategies for the prevention of APAPinduced hearing loss.

\section{Materials and methods Cell culture}

The HEI-OC1 cell line was kindly provided by Professor Federico Kalinec (House Ear Institute, Los Angeles, CA, USA). Mouse auditory cell lines (House Ear InstituteOrgan of Corti 1, HEI-OC1 cells) were cultured under permissive conditions $\left(33^{\circ} \mathrm{C}, 10 \% \mathrm{CO}_{2}\right)$ in high-glucose Dulbecco's modified Eagle's medium (DMEM; Gibco, \#11965092) containing 10\% fetal bovine serum (FBS; Gibco, \#10437028) without antibiotics ${ }^{30}$.

\section{Cell Counting Kit-8 (CCK-8) cell viability assay}

Cell viability was detected using a CCK- 8 assay kit (Dojindo Laboratories, Kumamoto, Japan). Briefly, HEIOC1 cells were trypsinized and collected. The cells were counted using an automated cell counter (Invitrogen Countess, Bothell, WA, USA), and the concentration was adjusted to $1.3 \times 10^{5}$ cells $/ \mathrm{ml}$. The cells were then seeded into a 96-well cell culture plate $(100 \mu \mathrm{l}$ per well) and incubated overnight for attachment. The cells were then treated with APAP (MedChemExpress, HY-66005) in a dose-dependent manner $(2,4,10,25,50 \mathrm{mM})$. After $24 \mathrm{~h}$, a CCK-8 assay was performed following the manufacturer's protocol. Subsequently, a microplate reader using the Gene5 software (BioTek Instruments, Winooski, VT, USA) measured the absorbance at $450 \mathrm{~nm}$. The average optical density (OD) value in the control cells was used as the baseline of $100 \%$ viability. The half-maximal inhibitory concentration (IC50) was calculated using the GraphPad Prism 6 software.

\section{Drug administration}

Chloroquine (CQ, Sigma-Aldrich, C6628) were used as the lysosomal inhibitor. The cell line was pretreated with CQ for $5 \mathrm{~h}$, and APAP was added for the following experiments. Bafilomycin A1 (Baf, Sigma-Aldrich, B1793) was used to block the fusion of autophagosomes with lysosomes. The cells were cotreated with Baf $(100 \mathrm{nM})$ and APAP for $24 \mathrm{~h}$. Acetylcysteine (NAC, MedChemExpress, HY-B0215) was used to inhibit the reactive oxygen 
species (ROS) accumulation. 4-Phenylbutyric acid (4PBA; Sigma-Aldrich, P21005) was used as an inhibitor of ER stress. After pretreated with $2 \mathrm{mM} \mathrm{NAC}$ or $1 \mathrm{mM} 4$ PBA for $4 \mathrm{~h}$, the cells were exposed to APAP for $24 \mathrm{~h}$.

\section{Crystal violet staining}

Cell viability was also determined by crystal violet staining as previously described ${ }^{31}$. In brief, HEI-OC1 cells were seeded into a 24-well cell culture plate. After the designated treatment, the cells were washed in ice-cold phosphate-buffered saline (PBS; Meilunbio, MA0015) and fixed in cooled methanol on ice for $10 \mathrm{~min}$. The cells were then stained with crystal violet solution $(0.5 \%$ crystal violet in $25 \%$ methanol) for $10 \mathrm{~min}$ at room temperature. The cells were washed with tap water and allowed to dry. Stained cells were then extracted with $1 \%$ sodium dodecyl sulfate. After solubilization, the $100 \mu \mathrm{l}$ per well dye extracts were measured at $570 \mathrm{~nm}$ using a microplate reader. The average OD value in the control cells was taken as $100 \%$ viability.

\section{Measurement of cell apoptosis by flow cytometry}

HEI-OC1 cells were seeded in a 6-well cell culture plate at a density of $4 \times 10^{5}$ cells $/ \mathrm{ml}(2 \mathrm{ml}$ per well) and incubated overnight. The cells were then treated with APAP at the concentration of either 10 or $20 \mathrm{mM}$ or with the vehicle solution ( $0.5 \%$ dimethyl sulfoxide (DMSO), containing a volume of DMSO equal to that used in $20 \mathrm{mM}$ APAP) for $24 \mathrm{~h}$. Apoptosis was quantified using an Annexin V-FITC Apoptosis Detection Kit I (BD Biosciences, 556547) according to the manufacturer's protocol. The stained cells were analyzed by a flow cytometer (FACSCanto II, BD Biosciences, San Jose, CA, USA).

\section{Mice}

The study involved the use of the C57BL/6J (B6) inbred mouse strain. B6 mice at 6-8 weeks of age were obtained from the Model Animal Research Center (Nanjing, China). In addition, green fluorescent protein (GFP)-LC3 mice (\#027139), obtained from the Jackson Laboratory, were used. Polymerase chain reaction (PCR) genotyping of the GFP-LC3 mice was performed according to the Jackson Laboratory's recommendation. The primers used for genotyping are shown in Table 1 (in the supplemental file). The Animal Use and Care Committee of Binzhou Medical University approved all mouse experimental protocols. Animals were randomly distributed over the different experimental conditions and blinding was applied during data acquisition and analysis when possible.

\section{Cochlear explant culture}

The cochlear explant culture procedures were performed as previously described ${ }^{32,33}$. In brief, mice at postnatal day 3 (P3) were euthanized after antisepsis with $70 \%$ ethanol, and the inner ears were removed. The cochleae were immersed in cold Hank's balanced salt solution (HBSS; Solarbio, \#H1045-500), and the organ of Corti and spiral ganglion neurons (SGNs) were carefully separated. The explants were placed onto a glassbottomed dish coated with tissue adhesion solution (Corning, \#354241), then washed by HBSS. Warmed culture medium (98\% DMEM, 1\% N-2, Thermo Fisher Scientific, \#17502-048) and 1\% ampicillin (Invitrogen, \#11593-027) were added directly onto the explants. The cochlear explants were incubated $\left(37^{\circ} \mathrm{C}, 5 \% \mathrm{CO}_{2}\right)$ for $10-16 \mathrm{~h}$, and then the final culture media (97\% DMEM, $1 \%$ FBS, $1 \% \mathrm{~N}-2$, and $1 \%$ ampicillin) was added to submerse the explants. APAP ( 5 or $10 \mathrm{mM}$ ) was added, and the tissue was incubated for $24 \mathrm{~h}$ to induce damage to the HCs of the explant-cultured cochleae.

\section{Mouse cochlear HC counts}

After the explant cultures were treated with 5 or $10 \mathrm{mM}$ APAP for $24 \mathrm{~h}$, the HCs were stained with phalloidinAlexa 488 (Invitrogen, A12379) to stain hair bundles and with 4,6-diamidino-2-phenylindole (DAPI; Invitrogen, D1306) to stain nuclei. The HCs were counted over a length of $150 \mu \mathrm{m}$ from the apical to the basal turns of the cochlea. For each experimental condition, at least four organ of Corti samples were analyzed.

\section{Immunofluorescence staining}

The cochlear explants were fixed for $30 \mathrm{~min}$ in $4 \%$ paraformaldehyde, washed 3 times for 5 min each with PBS, and blocked with 5\% goat serum in PBS for $1 \mathrm{~h}$. The tissues were then incubated with a primary antibody overnight at room temperature in blocking buffer. Then the tissues were washed 3 times for 5 min each with PBS and incubated with a secondary antibody in PBS for $1 \mathrm{~h}$. The tissues were washed 3 times in PBS and counterstained with DAPI. Finally, the tissues were mounted on slides in ProLong ${ }^{\mathrm{TM}}$ Gold Antifade Reagent (Cell Signaling Technology, \#9071). The following antibodies were used in this study: anti-cleaved caspase 3 (Cell Signaling Technology, \#9664, 1:400), anti-Myo7A antibody (Proteus BioSciences, \#25-6790, 1:150), anti-p62 (Abcam, ab56416, 1:50), anti-LAMP1 (Abcam, ab24170, 1:200), and anti-pS6 (Cell Signaling Technology, \#4858, 1:100). The stained tissues were imaged using a confocal microscope (LSM 880, Zeiss, Germany). The clock scan protocol was used for image analysis by ImageJ (National Institutes of Health, Bethesda, MA, USA) as previously described ${ }^{34}$.

\section{Quantification of the GFP-LC3 immunofluorescence signals in cultured cochlear explant tissues}

The GFP-LC3 fluorescent puncta were quantified as previously described ${ }^{13}$. After the indicated treatment of 
the cultured cochlear tissues, we counted the GFP-LC3fluorescent puncta in each $\mathrm{HC}$ and the number of $\mathrm{HCs}$ along the entire cochlear length to obtain the average number of GFP-LC3-fluorescent puncta per HC for each cochlea. At least three independent cochleae were counted.

\section{Western blotting}

HEI-OC1 cells were lysed with a RIPA lysis and extraction buffer (ThermoFisher Scientific, 89900) containing a protease and phosphatase inhibitor cocktail (Roche Diagnostics, Complete, 11697498001; PhosSTOP, 04906845001). The protein concentration was quantified using a BCA Protein Assay Kit (Takara, T9300A). Loading buffer (Takara, 9172) was added to the protein extract, and the sample was boiled for $5 \mathrm{~min}$ to denature the proteins. The protein extracts $(30-50 \mu \mathrm{g})$ were then separated on polyacrylamide gels and transferred to polyvinylidene fluoride membranes (Merck Millipore, IPVH00010) for $40 \mathrm{~min}$ at $15 \mathrm{~V}$ using a semidry transfer system (Bio-Rad, Hercules, CA, USA). The membranes were blocked with $5 \%$ nonfat dried milk in Tris-buffered saline containing $0.1 \%$ Tween 20 (TBS-T) for $1 \mathrm{~h}$ at room temperature. The membranes were then probed overnight at $4{ }^{\circ} \mathrm{C}$ with relevant primary antibodies: anti-cleaved caspase-3 (Cell Signaling Technology, \#9664), anti-Bcl-xL (Cell Signaling Technology, \#2764), anti-BiP (Proteintech, 11587-1-AP), anti-CHOP (Proteintech, 15204-1-AP), anti-ATF4 (Proteintech, 10835-1-AP), anti-ATF6 (ABclonal, A0202), antiXBP-1s (Cell Signaling Technology, \#12782), antiCaspase12 (Cell Signaling Technology, \#2202), anti-HO1 (Abcam, ab13248), anti-Nrf2 (Abcam, ab62352), antiLC3B (Novus Biologicals, NB100-2220), anti-p62 (Abcam, ab56416), anti-p-AMPK (Cell Signaling Technology, \#2531), anti-AMPK (Abcam, ab80039), anti-Hamartin/ TSC1 (Cell Signaling Technology, \#4906), anti-p-S6 (Ser235/236) (Cell Signaling Technology, \#4858), anti-S6 (Cell Signaling Technology, \#2217), anti-LAMP1 (Abcam, ab24170), anti-LAMP2 (Proteintech, 10397-1-AP), antiRab7 (Cell Signaling Technology, \#2094), anti-TFEB (Cell Signaling Technology, \#32361), anti-Atg7 (Cell Signaling Technology, \#8558), anti-Atg5 (Cell Signaling Technology, \# 12994), anti-Syntaxin17 (Proteintech, 17815-1-AP), antiVAMP8 (Proteintech, 15546-1-AP), anti-SNAP29 (Proteintech, 12704-1-AP), and anti- $\beta$-actin (Proteintech, 20536-1-AP). After washing with TBS-T, the membranes were probed again for $1 \mathrm{~h}$ at room temperature with a species-specific secondary antibody (anti-rabbit, Abcam ab6728 or anti-mouse, Abcam ab6721) coupled to horseradish peroxidase. The immunoreactive bands were detected using a Chemiluminescent HRP Substrate Kit (Bio-Rad, \#1705062, Clarity Max Western ECL Substrate) and visualized using the ChemiDoc XRS+ System (Bio$\mathrm{Rad})$. The intensities of the protein bands were measured and quantified using ImageJ, as described by Luke Miller (https://lukemiller.org/index.php/2010/11/analyzing-gelsand-western-blots-with-image-j/).

\section{MitoSOX staining}

MitoSOX ${ }^{\mathrm{TM}}$ Red (ThermoFisher, M36008) was used to analyze the mitochondrial ROS production. After the indicated treatment, the HEI-OC-1 cells were trypsinized and collected by centrifugation. The cells were resuspended in a solution containing $5 \mu \mathrm{M}$ MitoSOX reagent, incubated for $10 \mathrm{~min}$, washed with PBS, and analyzed by flow cytometry (FACSCanto II, BD Biosciences, San Jose, CA, USA). FlowJo V10 software was used to analyze the flow cytometric data. The explant-cultured tissues were also stained with $5 \mu \mathrm{M}$ MitoSOX after the indicated treatment. The samples were imaged using a confocal microscope (LSM 880, Zeiss, Germany).

\section{LysoTracker staining}

LysoTracker Red DND-99 (ThermoFisher, L7528) was used for the assessment of lysosome activity. This probe is highly selective for acidic organelles and is effective for labeling live cells ${ }^{35}$. After the indicated treatments, the HEI-OC1 cells were rinsed with PBS and stained with $100 \mathrm{nM}$ LysoTracker Red in a serum-free medium for $30 \mathrm{~min}$ in an incubator at $37^{\circ} \mathrm{C}$. The cells were then washed with PBS. Lysosome size and staining intensity were viewed using a confocal microscope (LSM 880, Zeiss, Germany). Lysosome sizes were measured using the ImageJ software. At least 30 cells of a random area were measured in each group. After the indicated treatment, the explant-cultured tissues were also stained with $100 \mathrm{nM}$ LysoTracker, and the samples were imaged using a confocal microscope (LSM 880, Zeiss, Germany).

\section{Magic Red staining}

Cathepsin B activity was measured using the Magic Red Cathepsin B Detection Kit (ImmunoChemistry Technologies, 937) as previously described ${ }^{12}$. Briefly, control or APAP-treated HEI-OC1 cells were cultured in confocal dishes (for microscopy) for different lengths of times, as indicated. The cells were then loaded with Magic Red Cathepsin B reagent for $1 \mathrm{~h}$, followed by washing twice with PBS. More than ten fluorescence images were taken using a confocal microscope and representative images are shown. ImageJ software was used to quantify the cathepsin B activity.

\section{Acridine orange staining}

Lysosomal V-ATPase activity was assessed with acridine orange staining. Acridine orange uptake represents lysosomal V-ATPase-driven pumping of hydrogen ions into the lysosomes ${ }^{36}$. After the indicated treatments, HEI-OC1 cells were washed with PBS and incubated with $1 \mu \mathrm{M}$ 
acridine orange (ThermoFisher, A1301) in the cultured medium for $30 \mathrm{~min}$ at $33^{\circ} \mathrm{C}$. The acridine orange was then removed, and the cells were examined under a confocal microscope (LSM 880, Zeiss, Germany). The cytoplasm and nuclei of stained cells showed bright green fluorescence, whereas the acidic autophagic vacuoles showed bright red fluorescence ${ }^{10,37}$.

\section{Plasmid transfection}

Autophagy flux was monitored by fluorescence microscopy of the distribution and alteration of mRFP-GFPLC3B fluorescence signals ${ }^{38}$. HEI-OC1 cells were seeded into glass-bottom dishes and transfected for $48 \mathrm{~h}$ with mRFP-GFP-LC3B plasmid (a gift from Dr. Yingyu Chen, Peking University, Beijing, China) using Lipofectamine 3000 transfection reagent (ThermoFisher Scientific, L3000015). After the designated treatments, the cells were fixed with $4 \%$ paraformaldehyde and examined under a confocal microscope. To quantify autophagic cells, GFPLC3 and mRFP-LC3 punctate dots were determined from triplicate samples by counting $>30$ cells.

\section{Transmission electron microscopy}

HEI-OC1 cells were fixed in $3 \%$ glutaraldehyde at $4{ }^{\circ} \mathrm{C}$ for $24 \mathrm{~h}$, postfixed in $1 \%$ osmium tetroxide for $1.5 \mathrm{~h}$, and dehydrated through a series of graded ethanol solutions. Fixed samples were embedded in Spurr's resin (Electron Microscopy Sciences, 14300) and thin sections $(80 \mathrm{~nm})$ were cut. The sections were stained with uranyl acetate and lead citrate and observed under a JEM-1400 transmission electron microscope (JEOL, Tokyo, Japan) at the Medicine Research Center, Binzhou Medical University, Yantai, China. The numbers of lysosomes and autophagic vacuoles were quantified in each sample for at least ten cells and confirmed by two additional repetitions of the experiments.

\section{Transfection with small interfering RNA (siRNA)}

The siRNA targeting mouse Atg5 or Atg7 and scrambled control siRNA were obtained from GenePharma (Shanghai). HEI-OC1 cells were transfected with $50 \mathrm{nM}$ siRNA or negative control siRNA using Lipofectamine 3000 Transfection Reagent (Invitrogen) according to the manufacturer's instructions. Seventy-two hours following transfection, the cells were exposed to $20 \mathrm{mM}$ APAP for $24 \mathrm{~h}$. The cells were analyzed by real-time cell analyzer (RTCA) or collected and processed for immunoblotting.

\section{Real-time cell analyzer}

Cytotoxicity was monitored by the xCELLigence RTCA DP system (ACEA Biosciences, USA) as previously described $^{39}$. First, the background of the E-plates was determined in $50 \mu \mathrm{l}$ of medium, and $100 \mu \mathrm{l}$ of the HEIOC1 cell suspension was added $\left(1.3 \times 10^{4}\right.$ cells per well).
Cells were incubated for $30 \mathrm{~min}$ at room temperature, and E-plates were placed into the RTCA station. Cells were grown for at least $24 \mathrm{~h}$, with impedance being measured every $15 \mathrm{~min}$. After the designated treatments, cells were monitored again every $15 \mathrm{~min}$ until the end of the experiment. The electronic readout, cell-sensor impedance induced by adherent cells to the electron flow, is displayed as an arbitrary unit, known as the cell index. The normalized cell index was calculated by the RTCA software at the selected normalization time point, which was chosen as the time immediately before the addition of treated drugs. Each treatment was performed in triplicate.

\section{Statistical analysis}

Each experiment was repeated at least three times. No samples or animals were excluded from the analysis. All data are presented as the mean \pm SEM. Microsoft Excel and GraphPad Prism 6 software were used for data analysis. Unpaired Student's $t$ test was used to determine statistical significance when comparing two groups, and one-way analysis of variance (ANOVA) was used when comparing more than two groups. A value of $P<0.05$ was considered statistically significant.

\section{Results \\ APAP induces apoptotic cell death in HEI-OC1 cells and cochlear HCs}

APAP-induced ototoxicity has been linked to cell death $^{29}$. In this study, we examined the underlying mechanisms for APAP-induced auditory cell death. First, HEI-OC1 cells were exposed to APAP, and the cell viability was examined using CCK- 8 and crystal violet staining assays. The CCK- 8 assay revealed that APAP exposure decreased the cell viability in a dose-dependent manner at $24 \mathrm{~h}$ after treatment (Fig. 1a). Because the calculated IC50 of APAP was $19.24 \mathrm{mM}$ (Fig. 1b), we used $20 \mathrm{mM}$ APAP as the treatment concentration for the subsequent HEI-OC1 cell experiments. Crystal violet staining revealed significant cell shrinkage and decreased cell numbers after APAP treatment (Fig. 1c, d). Flow cytometry showed that APAP induced HEI-OC1 cell death via apoptosis (Fig. 1e). The proportions of apoptotic cells at both the early $\left(\mathrm{AnxV}^{+} / \mathrm{PI}^{-}\right)$and the late phase $\left(A n x V^{+} / \mathrm{PI}^{+}\right)$after APAP treatment were significantly increased compared with the control cells (Fig. 1f, g). We also examined the expression of apoptosis-associated proteins (Fig. 1h). Western blotting showed that the protein expression of cleaved caspase 3 (CASP3) in HEIOC1 cells was significantly increased and the protein expression of Bcl-xl was significantly decreased after $24 \mathrm{~h}$ of $20 \mathrm{mM}$ APAP treatment compared with the control cells.

Next, we dissected the cochleae from postnatal day 3 (P3) mice and cultured the organ of Corti with 5 or 


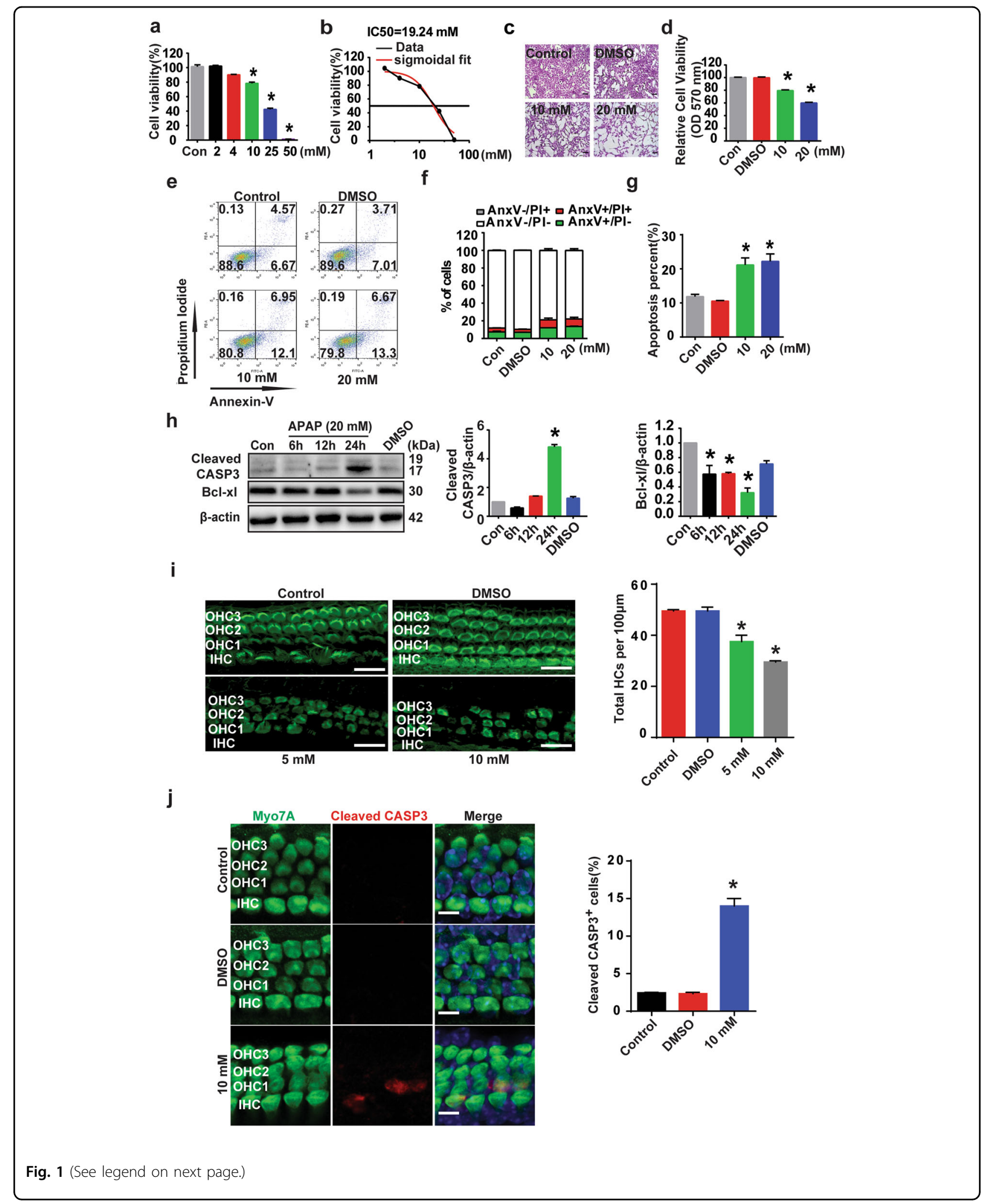


(see figure on previous page)

Fig. 1 APAP induced apoptotic cell death in HEI-OC1 cells and cochlear HCs. a HEI-OC1 cells were treated with APAP $(2,4,10,25,50$ mM) for $24 \mathrm{~h}$. The cell viability was detected by CCK-8 assay. ${ }^{*} P<0.05$ vs. control cells (Con). b The IC50 of APAP treatment was $19.24 \mathrm{mM}$ at $24 \mathrm{~h}$. IC50 with the curve fit line and the IC50 value are illustrated. c The cell viability was also determined by the crystal violet assay. HEl-OC1 cells were treated with $\operatorname{APAP}(10,20 \mathrm{mM})$ or vehicle (1\% DMSO with the volume equal to that used in $20 \mathrm{mM}$ APAP) for $24 \mathrm{~h}$. The cells were then stained with crystal violet, and morphological alterations were observed under a light microscope. Scale bar $=0.86 \mathrm{~mm}$. $\mathbf{d}$ The quantitative bar graph shows the relative density of cells from the crystal violet assay. ${ }^{*} P<0.05$ vs. control cells. e HEI-OC1 cells were treated with 1\% DMSO or APAP $(10,20$ mM). The percentage of apoptotic cells was determined by flow cytometry using annexin $V$ and PI labeling. The flow cytometric plots are representative of four experiments; mean \pm SEM are shown in the bar plots $(\mathbf{f}), \mathrm{AnxV}^{+} / \mathrm{PI}^{-}$(green bar; early apoptotic; lower right quadrant), $\mathrm{AnxV}^{+} / \mathrm{Pl}^{+}$(red bar; late apoptotic; upper

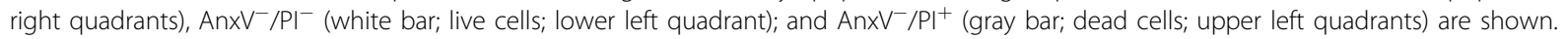
$\mathbf{g}$ Quantification of the proportion of apoptotic cells (early and late apoptotic cells) after APAP treatment from the flow cytometric data. ${ }^{*} P<0.05$ vs. control cells. $\mathbf{h} \mathrm{HEl}-\mathrm{OC} 1$ cells were treated with $20 \mathrm{mM}$ APAP for a designated period $(6,12$, or $24 \mathrm{~h}$ ) or vehicle (1\% DMSO) for $24 \mathrm{~h}$. The protein expression of cleaved caspase-3 and Bcl-xl was detected by western blotting. The right two panels show the results of densitometric analysis. ${ }^{*} P<$ 0.05 vs. control cells. $\mathbf{i}$ The cochlear explants treated with vehicle ( $0.5 \%$ DMSO) and $5 \mathrm{mM}$ or $10 \mathrm{mM}$ APAP for $24 \mathrm{~h}$ were stained for F-actin with phalloidin (green fluorescence). The images are representatives of four individual preparations. Confocal images were taken from the middle turn of the cochlea. Scale bar $=10 \mu \mathrm{m}$. OHC1, OHC2, and OHC3 represent the first, second, and third row of outer HCs, respectively; IHC inner HCs. The mean percentages of $\mathrm{OHC}$ loss are shown on the bar graph. Data are presented as the mean \pm SEM $\left(n=3\right.$ mice per group). ${ }^{*} P<0.05$ vs. control group. j The cochlear explants treated with vehicle (0.5\% DMSO) or $10 \mathrm{mM} \mathrm{APAP}$ for $24 \mathrm{~h}$. Apoptotic HCs were detected with a caspase 3 (CASP3) staining assay. Apoptotic cells were seen as caspase3-positive cells in the middle turns of cochlear explants from the APAP-treated groups. Scale bar $=5 \mu$ m. The right panel shows the comparison of the numbers of positive cells between the treated and control groups. ${ }^{*} P<0.05 \mathrm{vs}$. control group.

$10 \mathrm{mM}$ APAP for $24 \mathrm{~h}$. The mice cochlear explants in the absence of APAP maintained their normal morphology at $24 \mathrm{~h}$ (Fig. 1i). In contrast, outer $\mathrm{HC}$ loss began from the middle segment of the organ of Corti after $24 \mathrm{~h}$ of incubation with $5 \mathrm{mM}$ APAP and worsened with the increase in the concentration to $10 \mathrm{mM}$ (close to the IC50). The morphology of the stereociliary bundles of outer HCs was significantly changed after APAP treatment. The cochlear explants may have been more sensitive to the APAP treatment than the HEI-OC1 cells. Therefore, we used $10 \mathrm{mM}$ APAP as the treatment concentration of the subsequent mice cochlear explant experiments. Immunolabeling of cleaved CASP3, an apoptotic marker, also showed a significant increase in the number of CASP3positive $\mathrm{HCs}$ in the APAP-treated cochlear explants compared with the control group (Fig. 1j). These results demonstrated that APAP induces apoptotic cell death in HEI-OC1 cells and cochlear HCs.

\section{APAP induces oxidative stress in HEI-OC1 cells and cochlear HCs}

The cytotoxic effects of APAP might be associated with oxidative stress in HEI-OC1 cells because APAP significantly increased the production of ROS in these cells $^{29}$. We used a mitochondrial superoxide indicator, MitoSOX, to detect the mitochondrial ROS level in APAP-treated HEI-OC1 cells. The mitochondrial ROS levels significantly increased after APAP treatment compared with those of control groups at 12 and $24 \mathrm{~h}$ (Fig. 2a). Because ROS act as key mediators in APAP ototoxicity models, the antioxidant defense mechanism may be necessary to maintain normal cellular function. Thus, we examined the expression of oxidative stress-related proteins. Western blotting analysis revealed that Nrf-2 (NF-
E2-related factor-2) and its dependent protein, $\mathrm{HO}-1$ (heme oxygenase-1), both had higher expression levels when cells were exposed to APAP compared with control cells at 6 and $12 \mathrm{~h}$ (Fig. 2b). The Nrf-2 protein expression returned to the baseline at $24 \mathrm{~h}$ after APAP treatment, but HO-1 protein expression remained at a higher expression level than in control cells at $24 \mathrm{~h}$ after APAP treatment. The results suggest that the activation of the Nrf-2/HO1 signaling pathway may contribute to the APAP-induced oxidative stress. MitoSOX staining in the cochlear HCs also showed that the ROS level was significantly increased by APAP treatment (Fig. 2c). The results demonstrated that APAP induces oxidative stress in HEI-OC1 cells and in cochlear HCs.

\section{APAP induces ER stress in HEI-OC1 cells}

It has been reported that the cytotoxic effects of APAP on HEI-OC1 cells involve the activation of ER stress signaling by controlling the PERK-mediated branch of this signaling pathway ${ }^{29}$. Here we found that another branch of the ER stress response was also involved in APAP ototoxicity. We investigated the expression of several ER stress-inducible UPR proteins (Fig. S1a). APAP induced time-dependent increases in the protein expression levels of $\mathrm{BiP}$ (immunoglobulin-binding protein) and $\mathrm{CHOP}$ (CCAAT/enhancer-binding protein homologous protein) in HEI-OC1 cells. APAP treatment also increased the expression of ATF-4 protein at the early time point $(6 h)$, suggesting that APAP affects UPR events initiated by the stress sensor, PERK. To define the possible regulation of another stress sensor (IRE1 $\alpha$ ) by APAP, we examined the levels of IRE1 $\alpha$ 's downstream target X-box-binding protein-1 (XBP-1) in HEI-OC1 cells through western blotting and reverse transcription (RT)-PCR. The protein 
a
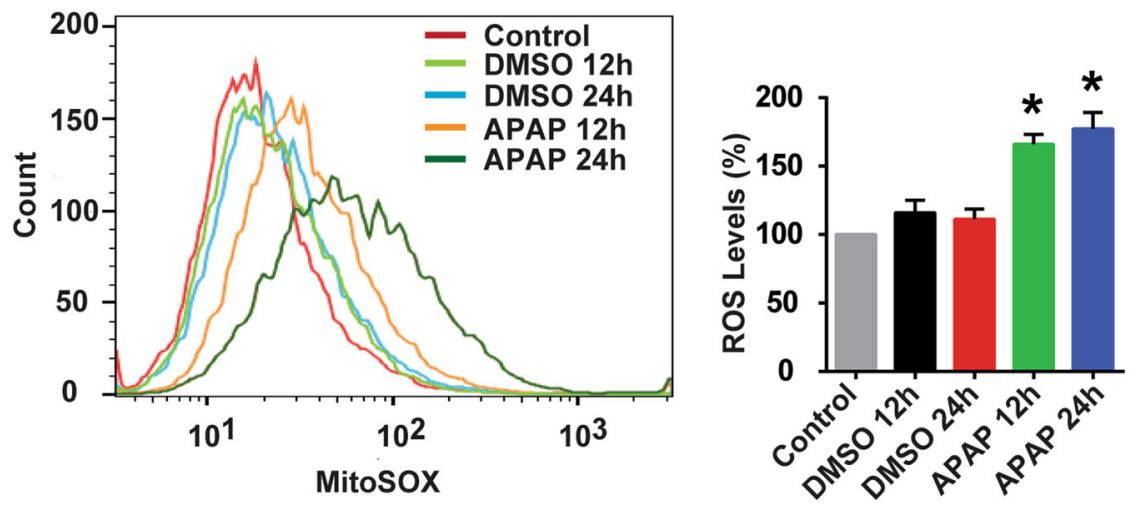

b
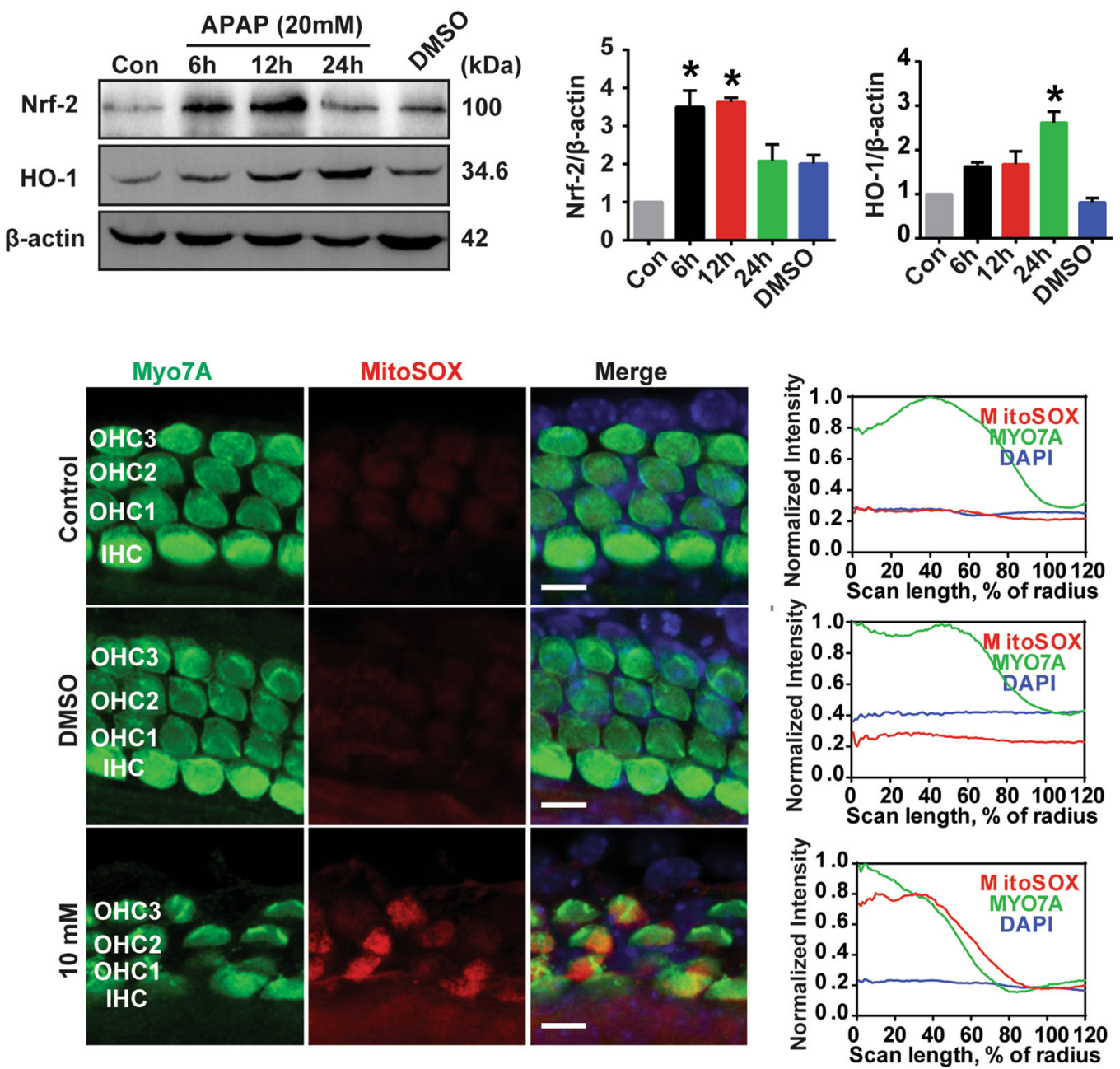

Fig. 2 APAP induces oxidative stress in HEI-OC1 cells and cochlear HCs. a HEI-OC1 cells were treated with 20 mM APAP or vehicle (1\% DMSO) for 12 or $24 \mathrm{~h}$. HEl-OC1 cells were then analyzed for mitochondrial superoxide generation by flow cytometry using MitoSOX Red. Representative line graphs are shown in the left panel. Quantification plots are shown in the right panel. The results were calculated as a percentage of normal control fluorescence intensity. Bars are the mean \pm SEM $(n=3)$. ${ }^{*} P<0.05$ vs. control cells. $\mathbf{b}$ HEl-OC1 cells were treated with 20 mM APAP for the indicated periods $(6,12$, or $24 \mathrm{~h}$ ) or vehicle (1\% DMSO) for $24 \mathrm{~h}$. The protein expression of $\mathrm{Nrf}-2, \mathrm{HO}-1$, and $\beta$-actin was measured by western blotting. The right panel shows the results of the densitometric analysis. ${ }^{*} P<0.05$ vs. control cells. $\mathbf{c}$ Immunofluorescence staining with Mito-SOX in the middle turn of the cochlea after treatment with $0.5 \%$ DMSO or $10 \mathrm{mM}$ APAP for $24 \mathrm{~h}$. Scale bar $=5 \mu \mathrm{m}$. Fluorescence intensities were quantified using the clock scan protocol in ImageJ. 
expression of spliced isoform XBP-1 (XBP-1s) was transiently enhanced at $6 \mathrm{~h}$ after APAP treatment but was subsequently decreased after $12 \mathrm{~h}$ of APAP treatment. Consistent with this finding, RT-PCR analysis revealed a significant increase in the levels of XBP-1 mRNA splicing in HEI-OC1 cells in the early time points after APAP treatment (Fig. S1b), indicating that APAP enhances the IRE1 $\alpha$ activity. Together, these results suggest that APAP induces the ER stress response in HEI-OC1 cells through PERK and IRE1 $\alpha$, two UPR branches.

\section{APAP impairs degradation of autophagosome cargo in HEI-OC1 cells and cochlear HCs}

In addition to induction of oxidative stress and ER stress, APAP treatment affects autophagy ${ }^{30}$. However, the role of autophagy in APAP-induced ototoxicity is not yet clear. In this study, we investigated the effect of APAP on this cellular degradation phenomenon. First, we determined the protein expression of an autophagosome marker, LC3, and an autolysosome marker, p62, in HEIOC1 cells with or without APAP treatment. A timedependent increase in LC3-II expression was observed in APAP-treated cells compared to control cells (Fig. 3a). LC3-II accumulation was increased at $24 \mathrm{~h}$ after APAP treatment. The p62 protein expression was also increased in a time-dependent manner after APAP treatment. To confirm autophagosome and autolysosome formation, we transfected HEI-OC1 cells with the GFP-LC3 plasmid and tandem mRFP-GFP-LC3 plasmid as reporter of autophagic flux ${ }^{40}$. We found that the numbers of GFP-LC3 puncta were significantly increased in HEI-OC1 cells after $24 \mathrm{~h}$ APAP exposure (Fig. S2). Autophagosomes that have not fused with lysosomes appear as yellow (mRFP and GFP) puncta, whereas autolysosomes appear as red (mRFP) puncta. The number of autolysosomes was markedly increased in the rapamycin-treated cells (positive autophagic control, rapamycin is a mammalian target of rapamycin (mTOR)-dependent autophagy activator) compared with control cells (Fig. 3b, c). The number of autophagosomes, but not autolysosomes, increased in APAP-treated cells compared with control cells. To further confirm the stage of inhibition on autophagy, we conducted autophagy flux assay. The blockage of autophagy flux with Baf showed that autophagosome synthesis is not increased with APAP treatment, because there is no difference in LC3-II protein level between Baf treatment and Baf plus APAP. On the other hand, it seems that the autophagy flux is reduced with APAP since the ratio of LC3-II APAP+Baf to LC3-II APAP is smaller than that of LC3-II Baf to LC3-II control, which explains the accumulation of LC3-II and p62 in the presence of APAP (Fig. 3e). Transmission electron microscopy was used to visualize the ultrastructures of autophagy organelles in HEI-OC1 cells (Fig. 3d). Taken together, the data demonstrate that APAP decreases the autolysosome formation in HEI-OC1 cells. Furthermore, these results indicate that APAP impairs the autophagy process, which might be associated with ER stress, oxidative stress, and cell death mechanism.

We also examined the autophagy process in mice cochlear explants after treatment with $10 \mathrm{mM}$ APAP for $24 \mathrm{~h}$. GFP-LC3 mice were used to examine the autophagosomes accumulation after APAP treatment. The cochleae were dissected from P3 GFP-LC3 mice and immunolabeled with an antibody for the $\mathrm{HC}$ marker myosin VIIA (Myo7A) after culturing the cochleae with $10 \mathrm{mM}$ APAP for $24 \mathrm{~h}$. The numbers of LC3 puncta were significantly higher in HCs after $24 \mathrm{~h}$ of APAP treatment compared with the controls (Fig. 3f, h). The cochlear explants were immunolabeled with an anti-p62 antibody after culturing the cochleae with $10 \mathrm{mM}$ APAP for $24 \mathrm{~h}$. The p62 protein expression was increased after APAP treatment (Fig. 3g, i-k). These results indicate that APAP induces autophagy impairment in mice cochlear explants. To investigate how the autophagy degradation process was impaired or blocked after APAP treatment, we assessed the protein expression of Rab7, Syntaxin 17, SNAP29, and VAMP8, which are necessary for the fusion of autophagosomes with lysosomes ${ }^{41}$. A reduction of SNAP29 levels but no detectable alteration in Rab7, Syntaxin 17, and VAMP8 levels was found in HEI-OC1 cells after treatment with APAP (Fig. S3). These results suggested that APAP may impair the fusion of autophagosomes with lysosomes by decreasing SNAP29.

\section{APAP-induced transient AMPK activation and mTORC1 suppression in HEI-OC1 cells and cochlear HCs}

The roles of AMPK and mTORC1 in autophagy could depend on cell types and metabolic conditions ${ }^{42}$. To find out whether AMPK and mTORC1 signaling are involved in APAP-induced autophagy impairment, the phosphorylation of AMPK and mTORC1 substrate, S6 ribosomal protein (S6) phosphorylation at 235/236 (p-S6), was examined. APAP treatment resulted in a significant time-dependent decrease in the levels of p-S6 compared with control cells (Fig. 4a). Immunoreactivity of p-S6 in the middle-turn outer HCs decreased in the APAP group, suggesting an inhibition of mTORC1 activity (Fig. 4b). AMPK activity was increased transiently as shown by increased phospho-AMPK level at 6 and $12 \mathrm{~h}$ after APAP treatment but decreased later (Fig. 4a). However, the inhibition of mTORC1 and increased activity of AMPK did not cause significant change in autophagosome formation as shown in autophagy flux assay. The meaning of APAP-induced changes of AMPK and mTORC1 signaling needs to be further studied by examining the phosphorylation and activities of other downstream factors, especially the ULK1 complex (ULK1PB1CC1/FIP200-ATG13-ATG101). 

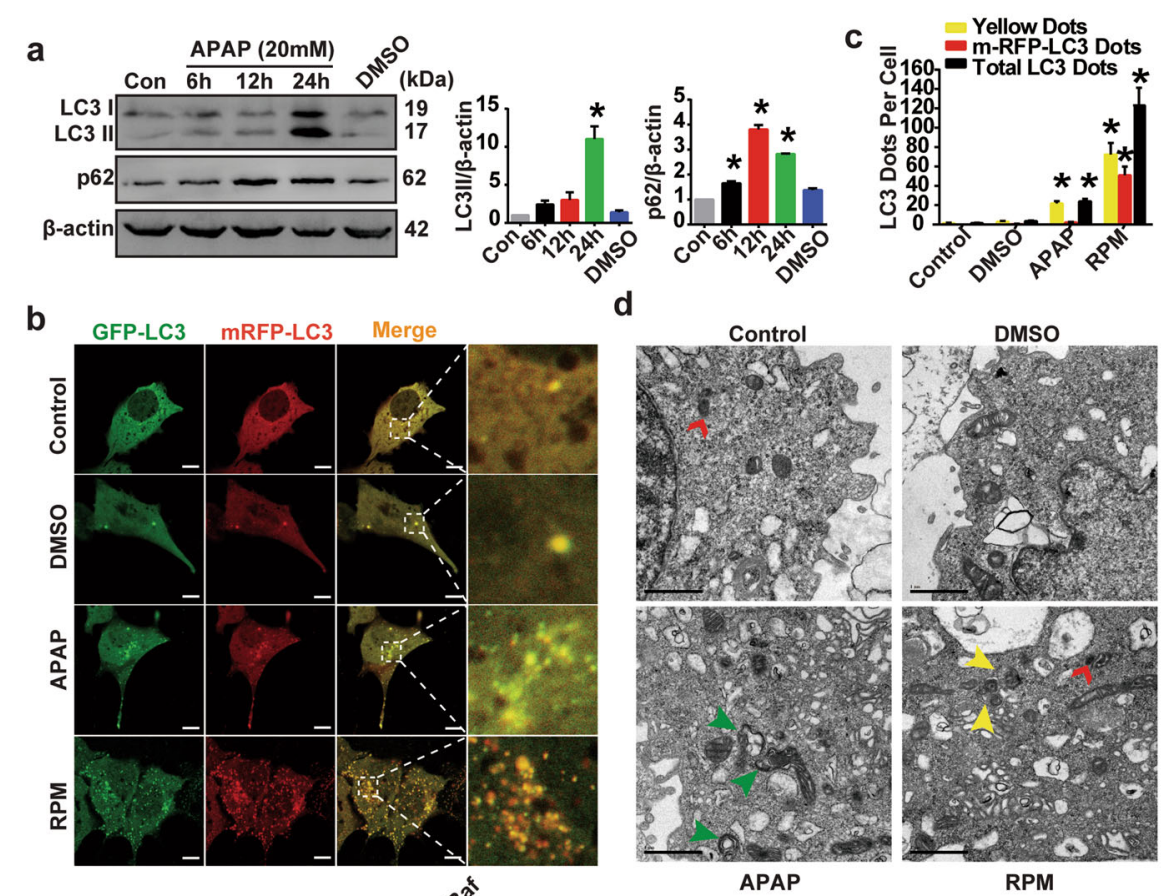

e
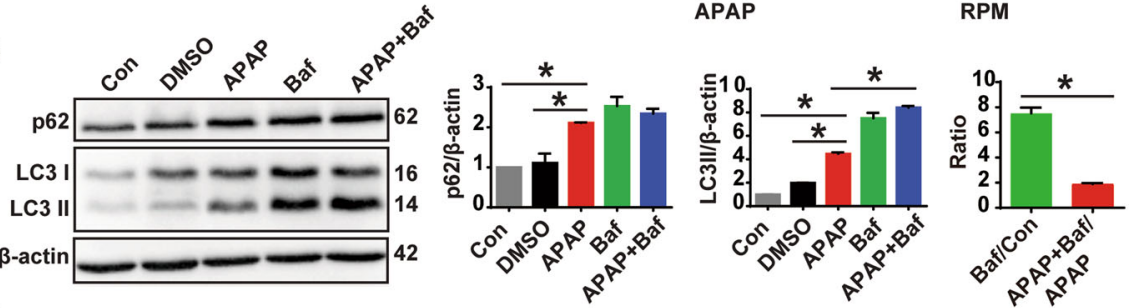

f

g
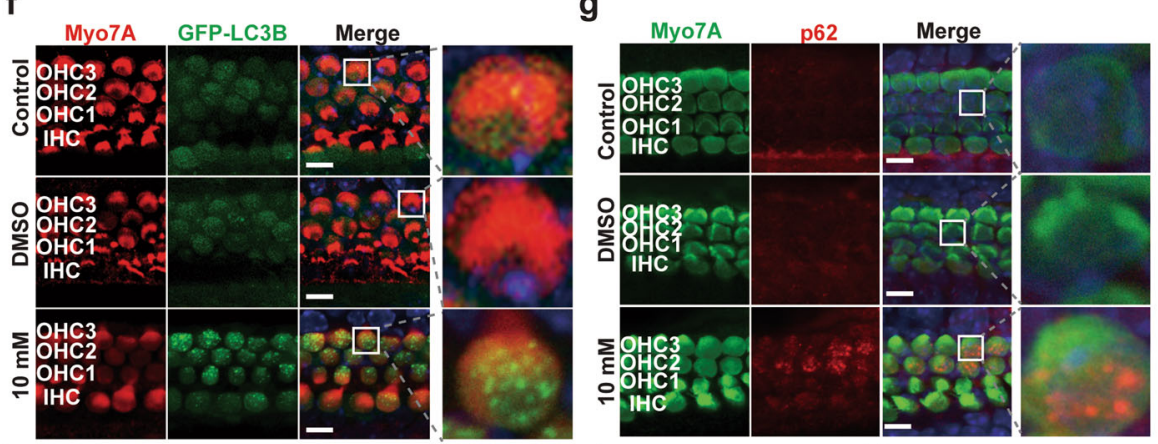

h
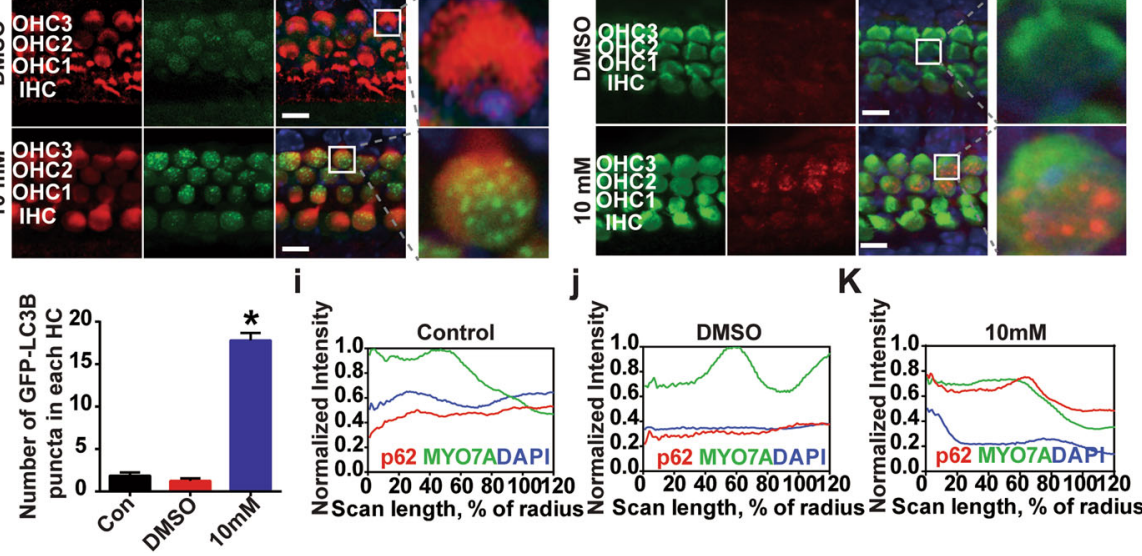

Fig. 3 (See legend on next page.) 
(see figure on previous page)

Fig. 3 APAP impairs degradation of autophagosome cargo in HEI-OC1 cells and cochlear HCs. a HEl-OC1 cells were treated with 20 mM APAP for the indicated periods $(6,12,24 \mathrm{~h})$ or vehicle $(1 \%$ DMSO) for $24 \mathrm{~h}$. The protein expression of LC3, p62, and $\beta$-actin was measured by western blotting. The right two panels show the results of the densitometric analysis ${ }^{*} P<0.05$ vs. control cells. $\mathbf{b} \mathrm{HEl}-\mathrm{OC} 1$ cells were transiently transfected with mRFP-GFP-LC3 plasmids for $48 \mathrm{~h}$, and then the cells were treated with vehicle (1\% DMSO), $20 \mathrm{mM}$ APAP, or $0.4 \mu \mathrm{M}$ rapamycin (RPM) for $24 \mathrm{~h}$. Red and green puncta were visualized by confocal microscopy. Enlarged images outlined by dashed lines illustrate punctate fluorescence. Scale bar $=$ $10 \mu \mathrm{m}$. c The bar graph shows the quantification of the ratio of mRFP-GFP-LC3 red to yellow puncta. ${ }^{*} P<0.05$ vs. control cells. $\mathbf{d}$ Lysosome and autophagic vacuoles (AVs) were analyzed by transmission electron microscopy in HEl-OC1 cells treated with DMSO, APAP, or RPM. Representative transmission electron micrographs of cell-in-cell structures showing autophagosomes (green arrowheads), lysosomes (red arrowheads), and autolysosomes (yellow arrowheads). Scale bar $=1 \mu \mathrm{m}$. e Western blot with anti-p62 and anti-LC3 antibodies after APAP and Baf treatment. ${ }^{*} P<0.05$. f Immunofluorescence staining with Myo7A antibody in cochlear explants from GFP-LC3 mice. Representative images are shown. Confocal images were taken from the middle turn. Enlarged images outlined by dashed lines illustrate punctate fluorescence. Scale bar $=5 \mu \mathrm{m}$. $\mathbf{g}$ Cochlear explants treated with vehicle $(0.5 \%$ DMSO) or $10 \mathrm{mM}$ APAP for $24 \mathrm{~h}$ were immunostained by Myo7A and p62 antibodies. The images are representative of four individual preparations. Confocal images were taken from the middle turn. Enlarged images outlined by white lines indicate p62 fluorescence in outer HCs. Scale bar $=5 \mu \mathrm{m}$. Fluorescence intensities were quantified using the clock scan protocol with ImageJ. The line plots are shown in i-k. h Quantification of the GFP-LC3 punctum number from $\mathbf{f} .{ }^{*} P<0.05$ vs. control cells. OHC1, OHC2, and OHC3 represent the first, second, and third row of outer HCs, respectively; $1 \mathrm{HC}$ inner HCs.

\section{APAP induces lysosome dysfunction in HEl-OC1 cells}

Lysosomal activity is important for the autophagy degradation process ${ }^{12,43}$. To investigate the APAP-induced autophagy impairment mechanism, we examined the function of lysosomes using a few lysosome indicators and the protein expression levels of key lysosome enzymes. First, using a lysosomal dye (LysoTracker Red) we found that the lysosomal fluorescence intensity did not increase in the APAP-treated HEI-OC1 cells compared with the control cells at 6 and $24 \mathrm{~h}$ (Fig. 5a). To verify the validity of our assay, we repeated the analysis in positive control samples. The lysosomal fluorescence intensity increased in the positive control cells (EBSS and rapamycin-treated cells). A lysosomal hydrolase, cathepsin B activity, was then measured using Magic Red reagent. We found that APAP-treated cells at the early treatment time did not have increased cathepsin B activity, but they showed increased activity at $24 \mathrm{~h}$ after APAP treatment (Fig. 5b). Consistent with these findings, staining with acridine orange, a lysosomal V-ATPase activity indicator, showed that APAP-treated cells did not have increased lysosomal V-ATPase activity at the early treatment time and that the activity increased only after a prolonged treatment $(24 \mathrm{~h})$ (Fig. 5c). The 3 staining methods revealed that the relative lysosome size increased significantly in the APAP-treated cells at $24 \mathrm{~h}$. These results suggest that treatment with APAP increases the number of enlarged acidic vesicles. We also measured the expression levels of lysosomalassociated membrane protein 1 (LAMP1) and LAMP2 (Fig. 5d). Both expression levels decreased at the early time point after APAP treatment and then slightly recovered with prolonged treatment. These findings suggested that the APAP-treated cells exhibited low lysosomal activity at the early time point, which was probably linked to the decreased autophagy-substrate clearance observed following APAP treatment.
We also examined transcription factor EB (TFEB), a master regulator of lysosomal biogenesis and autophagy ${ }^{44}$. The protein expression levels of TFEB significantly increased at the early treatment time but gradually returned to the baseline after a longer treatment time (Fig. S4a). HEI-OC1 cells transfected with TFEB plasmid showed stronger nuclear translocation of TFEB in the APAP-treated cells than in the control cells (Fig. S4b), suggesting that APAP induces lysosome biogenesis through TFEB activation. Moreover, we found enlarged vesicles accumulated around nuclei at the early time point after APAP treatment. This finding is consistent with the lysosome staining results in Fig. $5 \mathrm{a}-\mathrm{c}$ in which the relative lysosome size was increased after APAP treatment.

\section{APAP induces lysosome dysfunction in cochlear HCs}

The cochlear explants were immunolabeled with an anti-LAMP1 antibody after culturing the cochleae with $10 \mathrm{mM}$ APAP for 6 or $24 \mathrm{~h}$. LAMP1 protein expression decreased after APAP treatment in a time-dependent manner (Fig. 6a). Twenty-four hours after APAP treatment, the cytosolic LAMP1 diffused and distributed throughout the gap among HCs. The lysosomal fluorescence intensity (LysoTracker) decreased after APAP treatment (Fig. 6b). These results indicate that APAP may induce lysosomal impairment in mice cochlear explants.

\section{ROS signaling is involved in APAP-induced autophagy impairment}

Several lines of evidence indicate that ROS and ER stress are the upstream modulators of autophagy and signal to the autophagic machinery in the presence of stimuli $^{45-48}$. To investigate whether ROS is involved in APAP-induced autophagy impairment, we treated the HEI-OC1 cells with antioxidant NAC. Western blot results showed that, compared with the APAP-only group, 

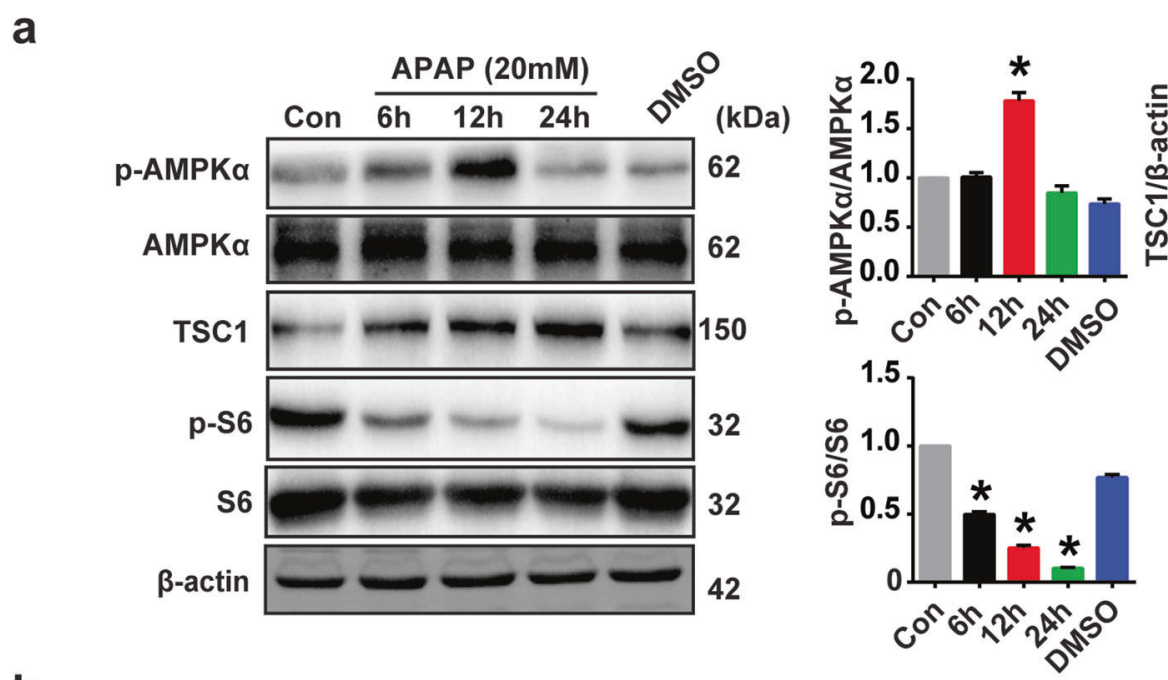

b
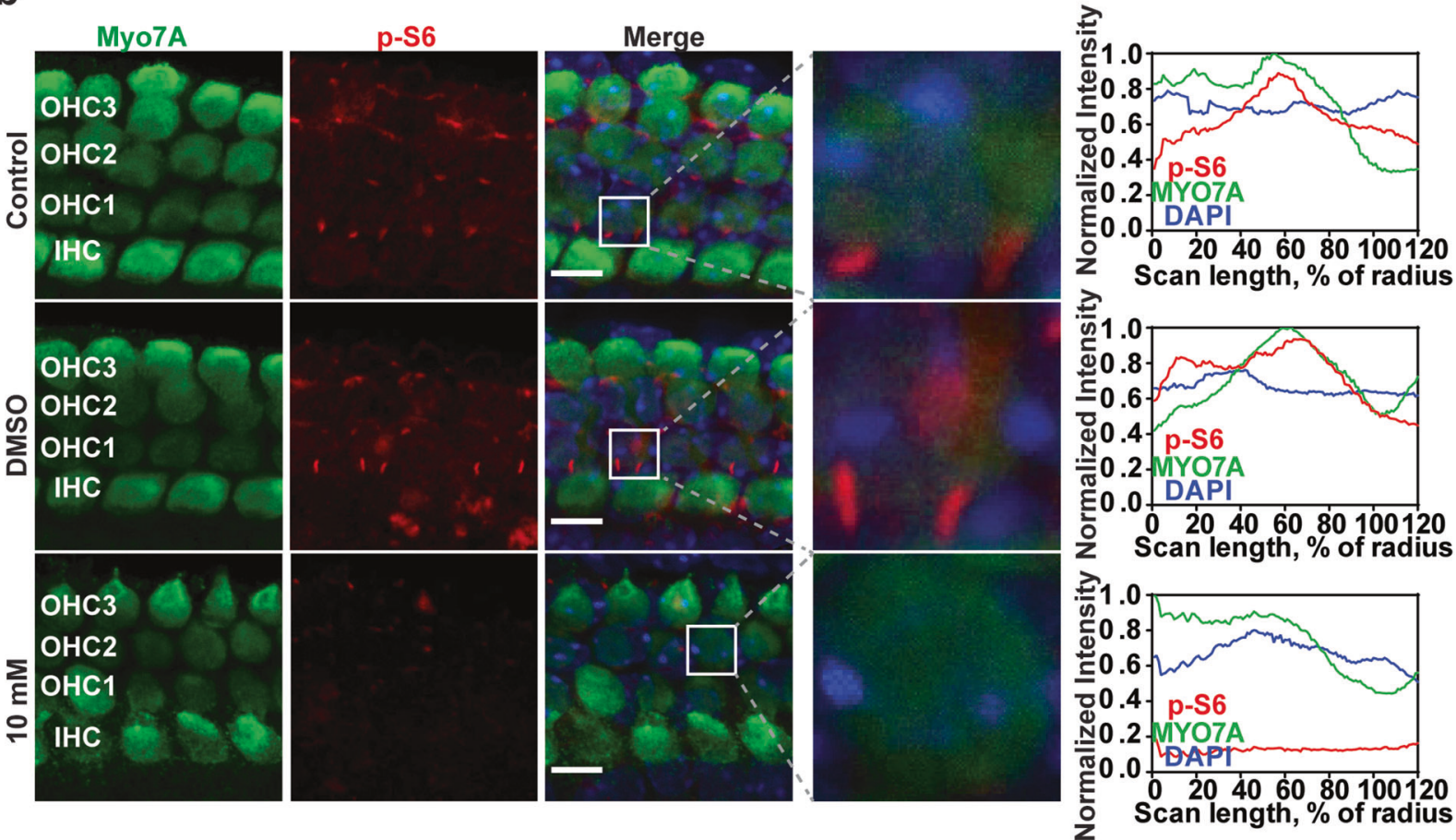

Fig. 4 APAP induces AMPK activation and mTORC1 suppression in HEI-OC1 cells and cochlear HCs. a The protein expression of p-AMPKa, AMPKa, TSC1, p-s6, s6, and $\beta$-actin was measured by western blotting. The three panels on the right show the results of densitometric analysis. ${ }^{*} P<0.05$ vs. control cells. b Cochlear explants treated with vehicle (0.5\% DMSO) or $10 \mathrm{mM}$ APAP for $24 \mathrm{~h}$ were immunostained by Myo7A and $\mathrm{p}$-s6 antibodies. The images are representative of four individual preparations. Confocal images were taken from the middle turn. Enlarged images outlined by white line indicate $p$-s6 fluorescence in outer HCs. Scale bar $=5 \mu \mathrm{m}$. Fluorescence intensities were quantified using the clock scan protocol in ImageJ.

the expression level of $\mathrm{HO}-1$ was significantly decreased in NAC+APAP group, which suggested that APAPinduced ROS level was decreased in the pretreatment with NAC (Fig. 7a). Meanwhile, we observed a reduction in the levels of p62 and LC3-II in NAC-treated cells compared with the APAP-only group, indicating that NAC treatment attenuated APAP-induced autophagy impairment
(Fig. 7a). Taken together, our results indicated the involvement of oxidative stress in APAP-induced autophagy impairment as well as apoptotic cell death. It is well known that ROS induces ER stress. We also observed a reduction of ER stress by NAC treatment (Fig. S5).

To understand the relationship between ER stress and autophagy in HEI-OC1 cells, we tested the effect of ER 


\section{a}

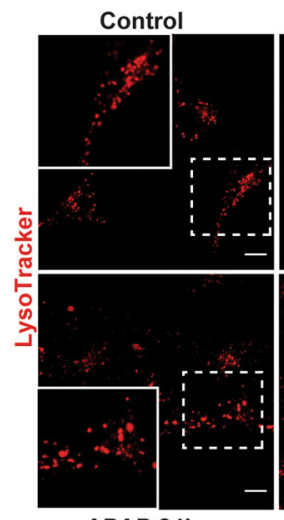

b

APAP 24h

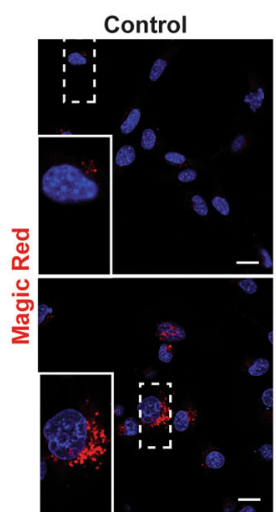

APAP 24h

C

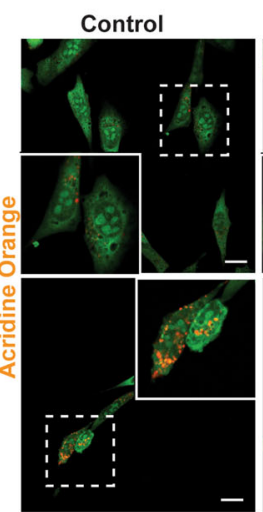

APAP 24h
DMSO

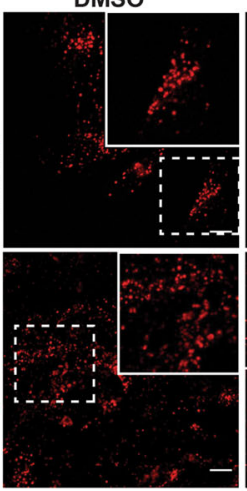

EBSS DMSO

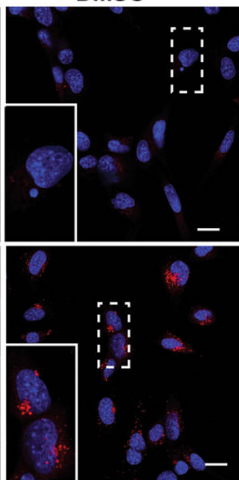

EBSS

DMSO

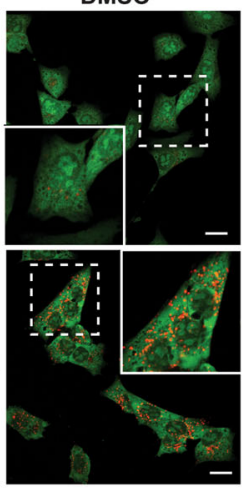

EBSS
APAP $6 \mathrm{~h}$
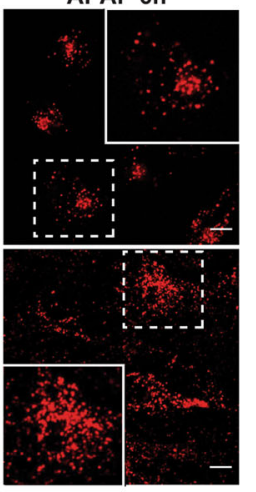

RPM

APAP $6 h$

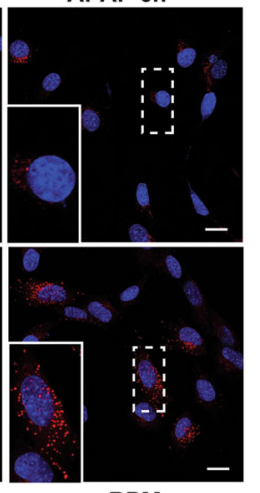

RPM

APAP $6 h$

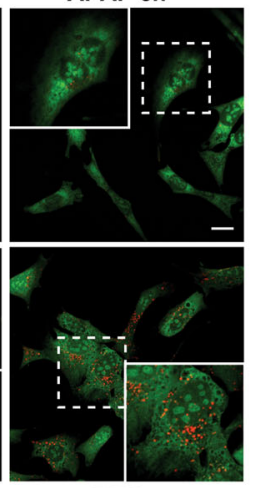

RPM
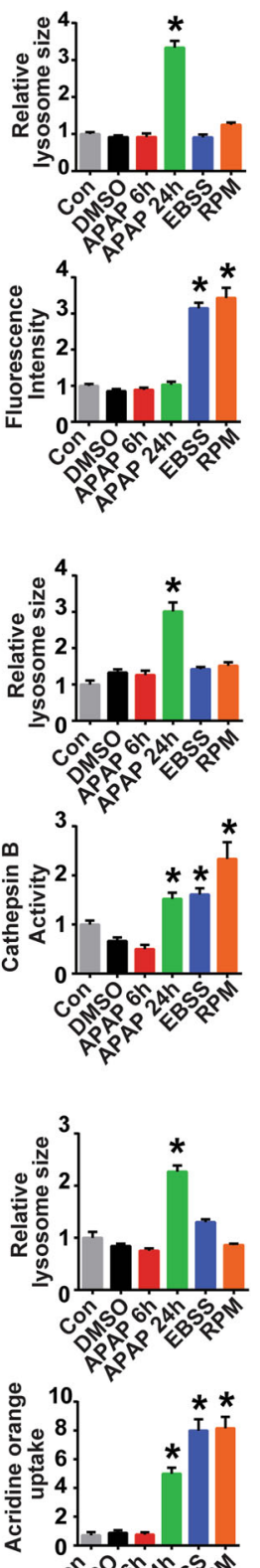

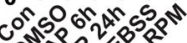

b?
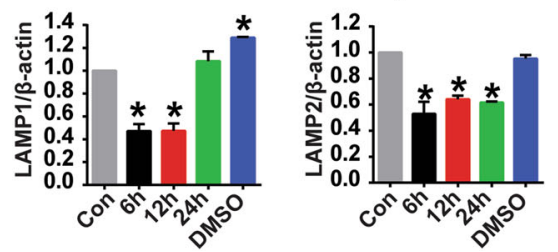

Fig. 5 APAP inhibits lysosome function in HEI-OC1 cells. a-c HEI-OC1 cells were treated with vehicle (1\% DMSO) or 20 mM APAP for 6 or $24 \mathrm{~h}$. The positive control cells were treated with EBSS for $2.5 \mathrm{~h}$ or with $0.4 \mu \mathrm{M}$ rapamycin (RPM) for $24 \mathrm{~h}$. The cells were exposed to $100 \mathrm{nM}$ LysoTracker or Magic Red reagent (b) or $1 \mathrm{mg} / \mathrm{ml}$ acridine orange (c). Images were taken by confocal microscopy. Representative images and enlarged images outlined by white lines are shown; scale bar $=5 \mu \mathrm{m}$. Relative lysosome sizes and fluorescence intensities were quantified using ImageJ. ${ }^{*} P<0.05$ vs. control cells. $\mathbf{d} \mathrm{HEI}-\mathrm{OC} 1$ cells were treated with vehicle (1\% DMSO) or $20 \mathrm{mM}$ APAP for the indicated periods $(6,12,24 \mathrm{~h})$. Protein expression of LAMP1, LAMP2, and $\beta$-actin was measured by western blotting. The right panel shows the results of the densitometric analysis. ${ }^{*} P<0.05$ vs. control cells. 
a

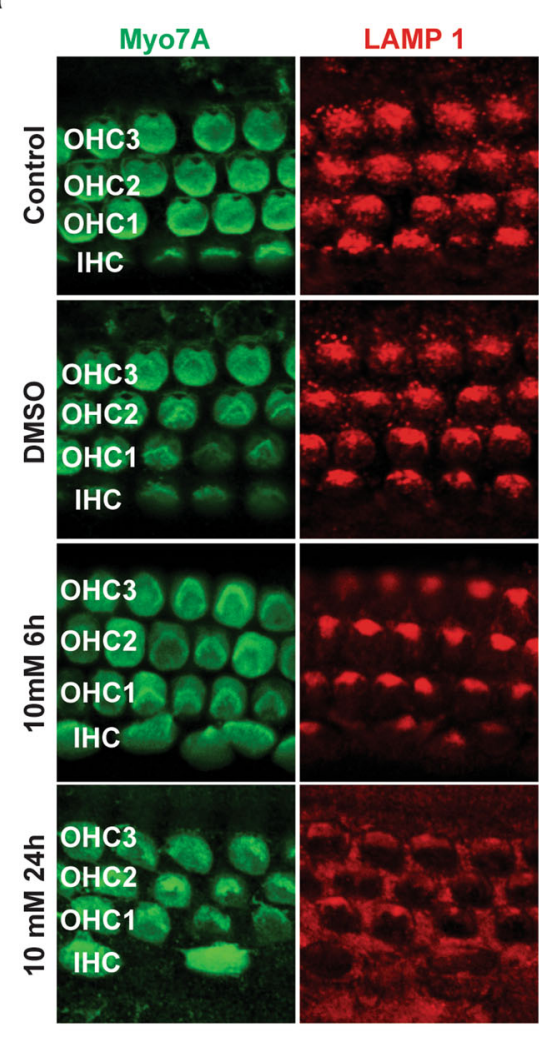

b

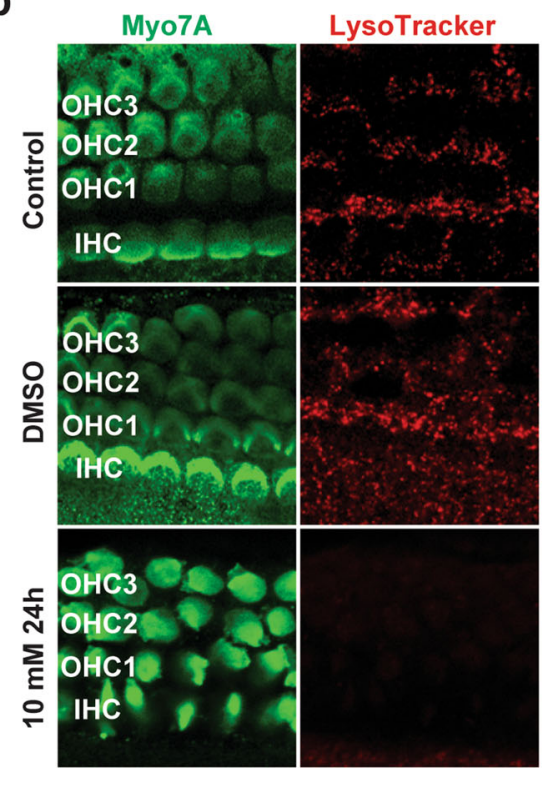

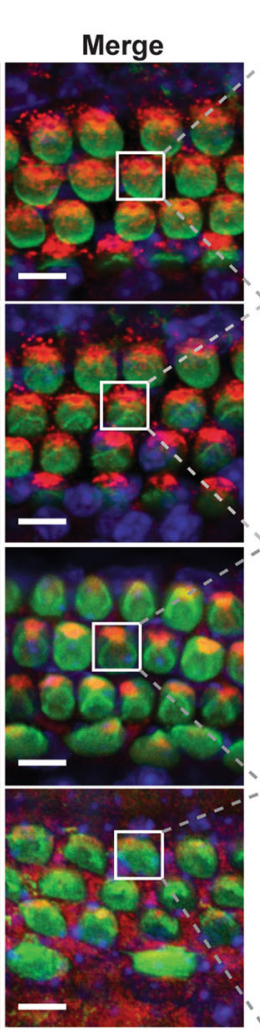
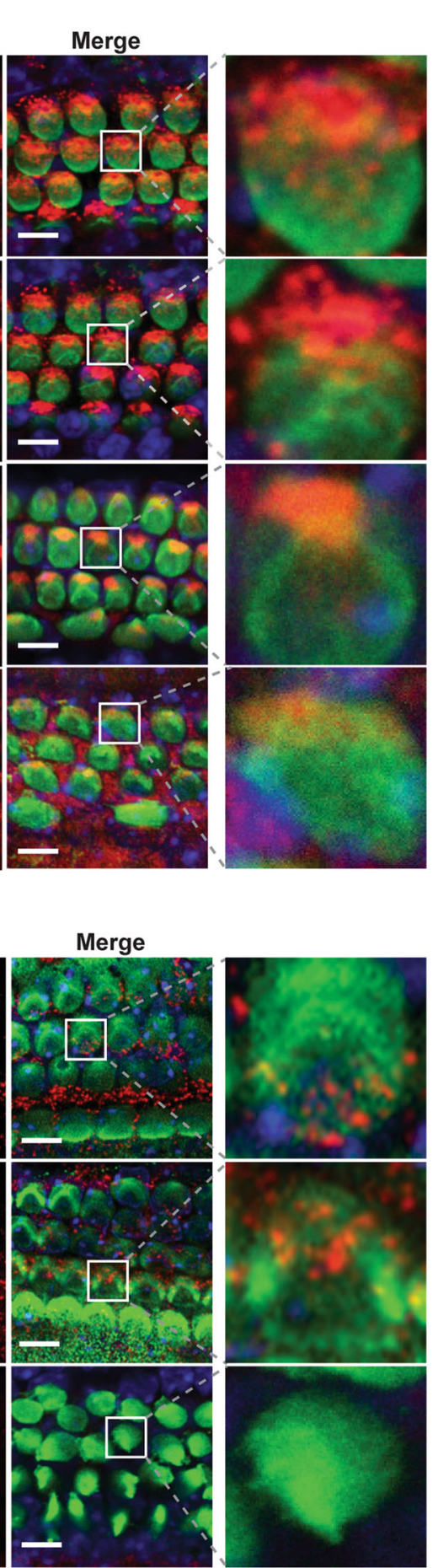

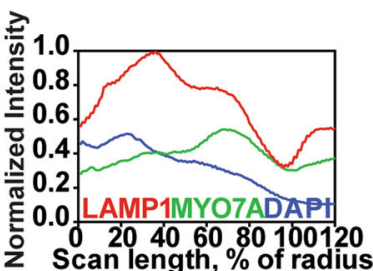

离1.0

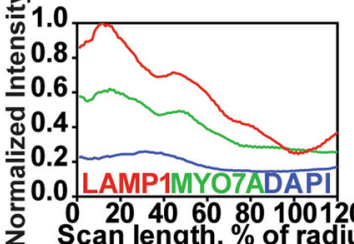

ㄴ. Scan length, \% of radius

$\geq 10$

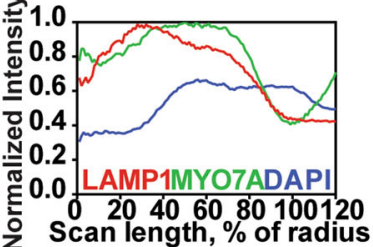

要1.0

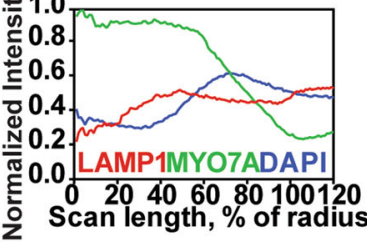

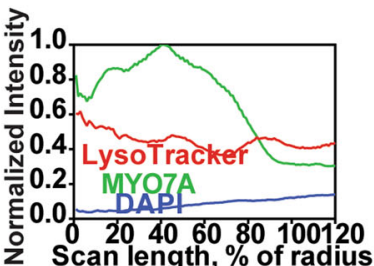

ते 1.0

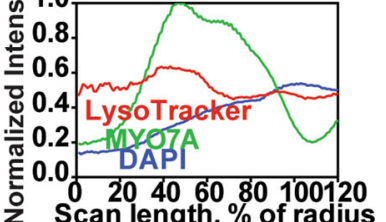

Scan length, \% of radius

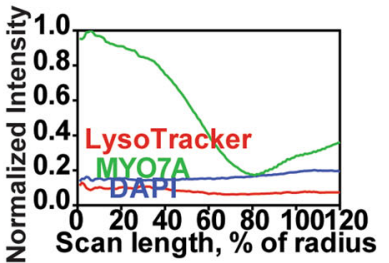

Fig. 6 APAP inhibits lysosome function in cochlear HCs. a The cochlear explants treated with vehicle (0.5\% DMSO) or $10 \mathrm{mM}$ APAP for 6 or $24 \mathrm{~h}$ were immunostained for Myo7A (green fluorescence) and LAMP1 (red fluorescence). Enlarged images outlined by white lines indicate LAMP1 fluorescence in outer HCs. The images are representative of four individual preparations. Confocal images were taken from the middle turn. Scale bar $=5 \mu \mathrm{m}$. Fluorescence intensities were quantified using the clock scan protocol in ImageJ. b The cochlear explants treated with vehicle (0.5\% DMSO) or $10 \mathrm{mM}$ APAP for $24 \mathrm{~h}$ were stained by LysoTracker Red. Enlarged images outlined by white lines indicate LysoTracker fluorescence in outer HCs. The images are representative of four individual preparations. Confocal images were taken from the middle turn. Scale bar $=5 \mu \mathrm{m}$. Fluorescence intensities were quantified using the clock scan protocol by ImageJ. $\mathrm{OHC1}, \mathrm{OHC2}$, and $\mathrm{OHC} 3$ represent the first, second, and third row of outer $\mathrm{HCS}$, respectively; $I H C$ inner $\mathrm{HCS}$. 

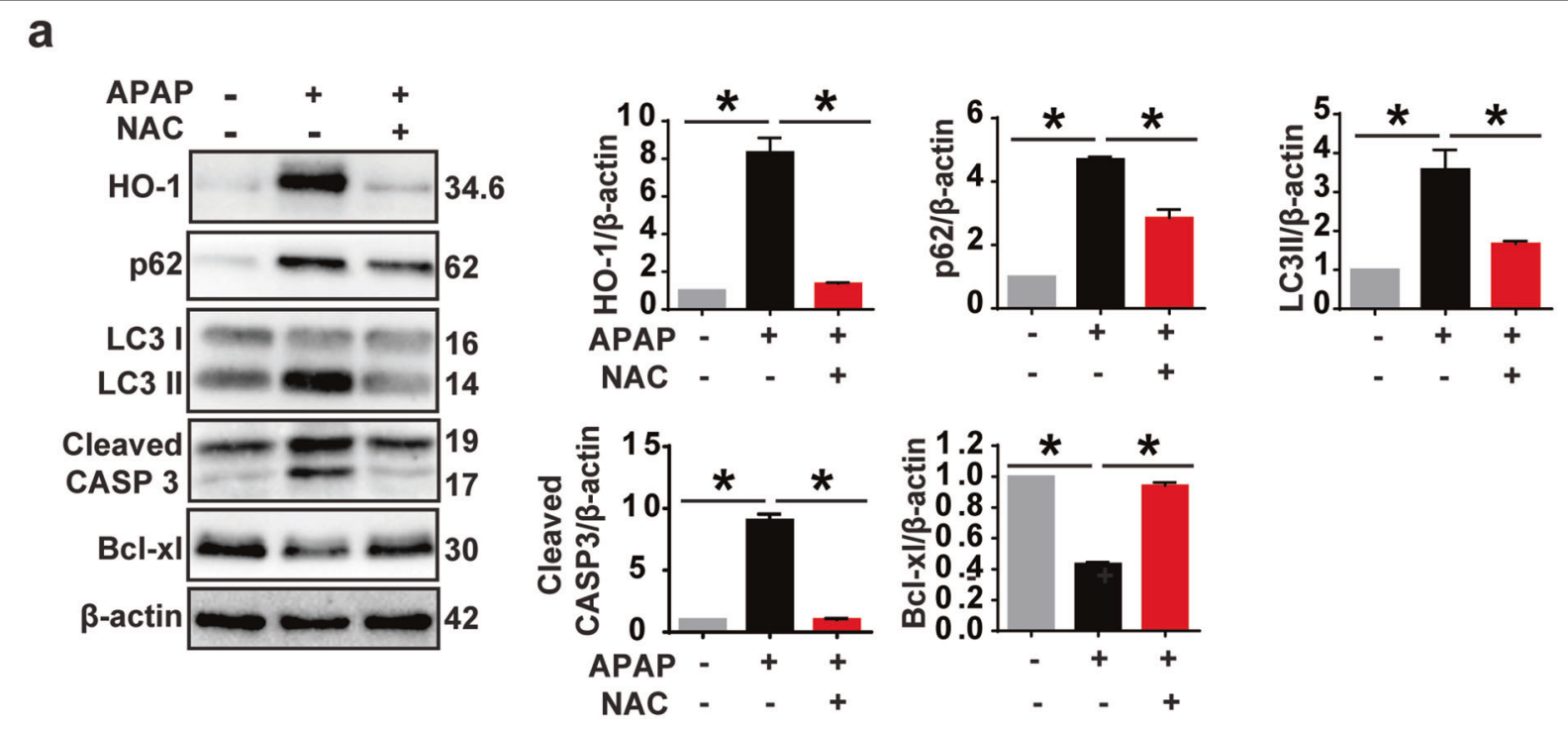

b
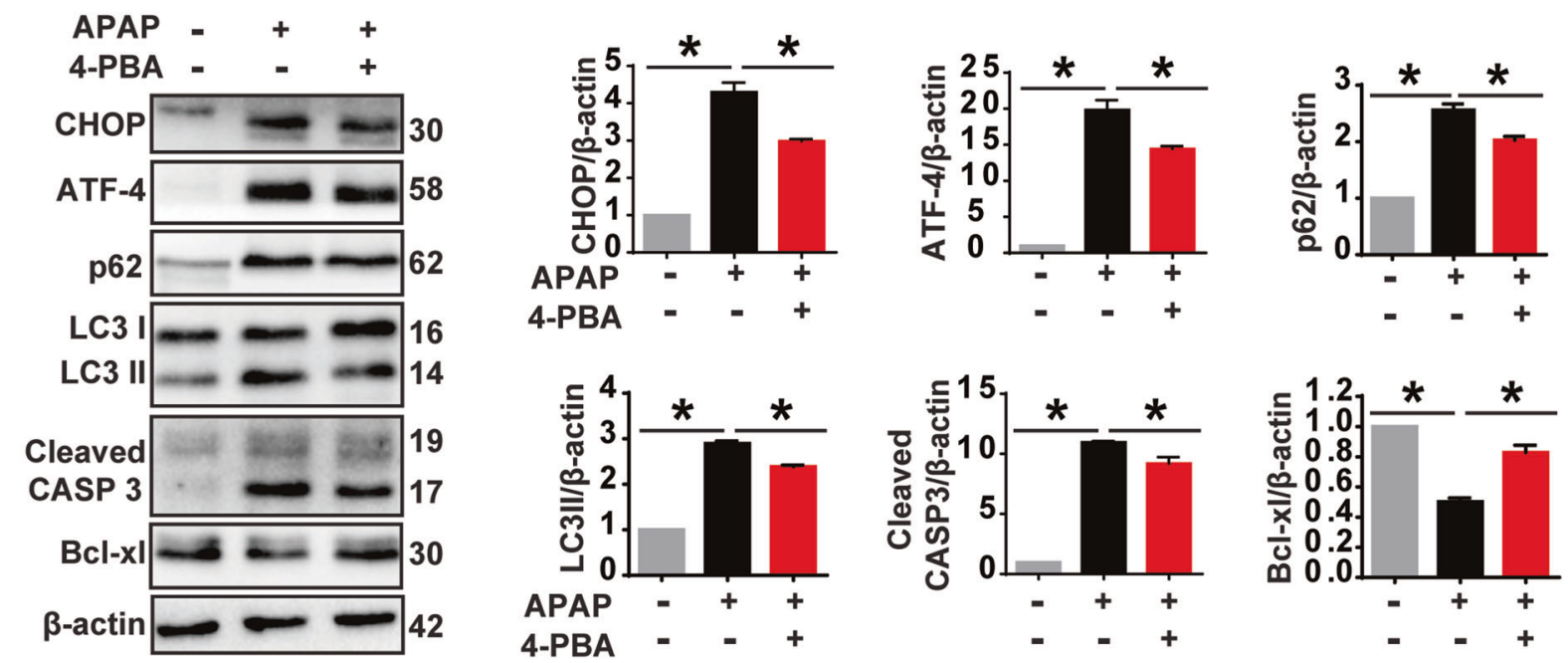

Fig. 7 Inhibition of ER stress or oxidative stress in HEI-OC1 cells affect APAP-induced autophagy process and cell viability. a Western blots with anti-HO-1, anti-p62, anti-LC3, anti-cleaved CASP3, and anti-BCl-xl antibodies after APAP and antioxidant treatment (NAC). The four panels on the right show the results of densitometric analysis. ${ }^{*} P<0.05$. $\mathbf{b}$ Immunoblot analysis of APAP-induced change in the CHOP, ATF-4, p62, LC3, cleaved CASP3, and $\mathrm{BCl}-\mathrm{xl}$ in the presence of the ER stress inhibitor 4-PBA. The graphs show quantification of band intensities. ${ }^{*} P<0.05$.

stress inhibitor 4-PBA on APAP-induced autophagy impairment and apoptotic cell death. Western blot results show a decrease in CHOP and ATF-4 in the 4-PBA +APAP group compared with APAP treatment alone, indicating that 4-PBA treatment partially suppressed APAP-induced ER stress (Fig. 7b). LC3-II and p62 protein levels were also slightly decreased in 4-PBA+APAP treatment compared to APAP alone, indicating 4-PBA has weak effect on the alleviation of APAP-induced autophagy impairment (Fig. 7b). Immunoblot analysis of Bcl-xl and cleaved CASP3 showed that 4-PBA treatment slightly attenuated APAP-induced apoptotic cell death (Fig. 7b).
Weak effect was also seen in apoptotic signaling proteins but no effect was seen on cell survival (data not shown). This result suggested that ER stress has a weak, if any, contribution to APAP-induced autophagy impairment, and intervention on ER stress signaling was not an effective way to attenuate APAP-induced cell death.

Inhibition of autophagy enhances APAP-induced ER stress, oxidative stress, and apoptotic cell death

To determine the role of autophagy in APAP-induced oxidative stress, ER stress, and cell death in HEI-OC1 cells, we tested the effects of knocking down autophagy 
genes ( $\operatorname{Atg} 5$ and $\operatorname{Atg} 7)$ and lysosomal inhibitor that disrupts the fusion of autophagosome and lysosome, in the presence of APAP, on ER stress, oxidative stress, and apoptotic cell death in HEI-OC1 cells. RTCA and immunoblot analysis of cleaved CASP3 showed that CQ + APAP aggravates apoptotic cell death compared to APAP alone group (Fig. 8a, d). CQ+APAP significantly increased LC3-II protein level compared with APAP alone group in HEI-OC1 cells (Fig. 8d). However, there was no significant change in the accumulation of p62 in the presence of CQ compared to the APAP alone group (Fig. 8d). We found that Atg5 knockdown significantly upregulated the expression of oxidative stress-related protein (HO-1) and ER stress-related protein (ATF-4, Bip) compared with the APAP-only group (Fig. 8e). The western blot results of $\operatorname{Atg} 7$ knockdown are similar to that of $\operatorname{Atg} 5$ (Fig. S6). These results suggested that loss of autophagy gene $\operatorname{Atg} 5$ or $\operatorname{Atg} 7$ induces oxidative stress and ER stress, indicating a feedback mechanism of autophagy on these processes. RTCA and immunoblot analysis of Bcl-xl and cleaved CASP3 showed that, compared with the APAPonly group, apoptotic cell death was significantly increased in the $\operatorname{Atg} 5$ siRNA+APAP and $\operatorname{Atg} 7$ siRNA +APAP groups (Fig. 8b, c, e). These results demonstrated that autophagy plays an important role in APAP-induced apoptotic cell death in HEI-OC1 cells after APAP injury.

\section{Discussion}

HCs in the cochlea play a critical role in converting mechanical sound waves into neural signals, and SGNs transmit these signals to the auditory cortex, resulting in hearing ${ }^{15,49,50}$; thus HCs and SGNs are critical for hearing ability. In the inner ear of mammals, $\mathrm{HCs}$ are vulnerable to multiple types of damage, and these cells lack the ability to regenerate. Therefore, dead HCs cannot be spontaneously regenerated ${ }^{51-56}$, which serves as the major cause of the hearing loss induced by gene mutation, noise, ototoxic drugs, inflammation, and aging ${ }^{15,57-60}$. In this study, we investigated the molecular mechanisms of APAP-induced ototoxicity using two in vitro models: HClike HEI-OC1 cells and whole-organ primary culture of the murine organ of Corti.

In the inner ear, apoptosis of HCs can be activated in response to various types of damage, including acoustic trauma, ototoxicity, inflammation, and aging ${ }^{61-65}$. In this study, we showed that APAP induced apoptotic cell death in HEI-OC1 cells and in mouse cochlear HCs. After treatment with APAP, the HEI-OC1 cell viability was markedly decreased, but the percentage of dead cells was not very high. These results may suggest that APAP exposure decreases cell division, a phenomenon that has been previously reported ${ }^{30}$.

Noise, ototoxic drugs, inflammation, and aging can all increase the accumulation of mitochondrial ROS, leading to mtDNA damage, oxidative stress, and ER stress in $\mathrm{HCs}$, causing HC apoptosis ${ }^{16,23,61,63}$. We observed that APAP induced oxidative stress, ER stress, and apoptosis in HEIOC1 cells and cultured cochlear explants, which is consistent with previous reports ${ }^{29,30}$. Kalinec et al. reported that the toxic effects of APAP in HEI-OC1 cells involve the induction of ER stress signaling by controlling the PERK-mediated branch of this signaling pathway ${ }^{29}$. They suggested that the IRE1 $\alpha$-mediated ER stress pathway is not involved in APAP or NAPQI cytotoxicity. They found that HEI-OC1 cells exposed to APAP showed no clear signals of XBP-1 splicing, the downstream of IRE1 $\alpha$ signaling. However, in this study, we showed that the IRE1 $\alpha$ mediated ER stress pathway is involved in APAP ototoxicity. The protein expression of XBP-1s was transiently enhanced at $6 \mathrm{~h}$, and the levels of XBP-1 mRNA splicing were increased in a time-dependent manner in the early time points after APAP treatment.

APAP-induced ototoxicity has been linked to autophagy $^{30}$. In this study, we found that treatment with APAP caused autophagy impairment as shown by the accumulation of p62. We continued to show that APAP-induced autophagy impairment was due to the reduced lysosomal activity but not formation of autophagosome. By applying LysoTracker, Magic Red, and acridine orange staining, we found that the accumulation of large liposomal vesicles increased significantly in APAP-treated cells at $24 \mathrm{~h}$. Similarly, a disruption in autophagy is also associated with the accumulation of large vesicular structures in the inner segments of cone photoreceptors, increases in enlarged acidic vesicles, and abnormal late endosomes in zebrafish lacking polyphosphoinositide phosphatase synaptojanin $1^{66}$. Researchers have demonstrated that lysosomal dysfunction is more directly responsible for autophagy impairment ${ }^{12}$. We also found that the protein expression of lysosomal proteins, LAMP1 and LAMP2, was decreased after APAP treatment in both HEI-OC1 cells and cochlear HCs. We further showed that inhibition of lysosomal activity by CQ further aggravated autophagy impairment and increased apoptotic cell death. Therefore, we suggest that APAP may impair degradation of autophagosome cargo through lysosome dysfunction in HEIOC1 cells and cochlear HCs.

In this study, we investigated three cell-signaling pathways (oxidative stress, ER stress, and autophagy) in the context of APAP-induced ototoxicity. We suggest that the three cell-signaling pathways are all involved in APAPinduced ototoxicity. It has been reported that ROS-induced mitochondrial damage may be an important upstream activator of mitophagy, a process for the selective autophagic degradation of mitochondria ${ }^{67}$. In this study, we found that ROS signaling is involved in APAP-induced autophagy impairment because antioxidant NAC treatment partially alleviated APAP-induced autophagy impairment as 


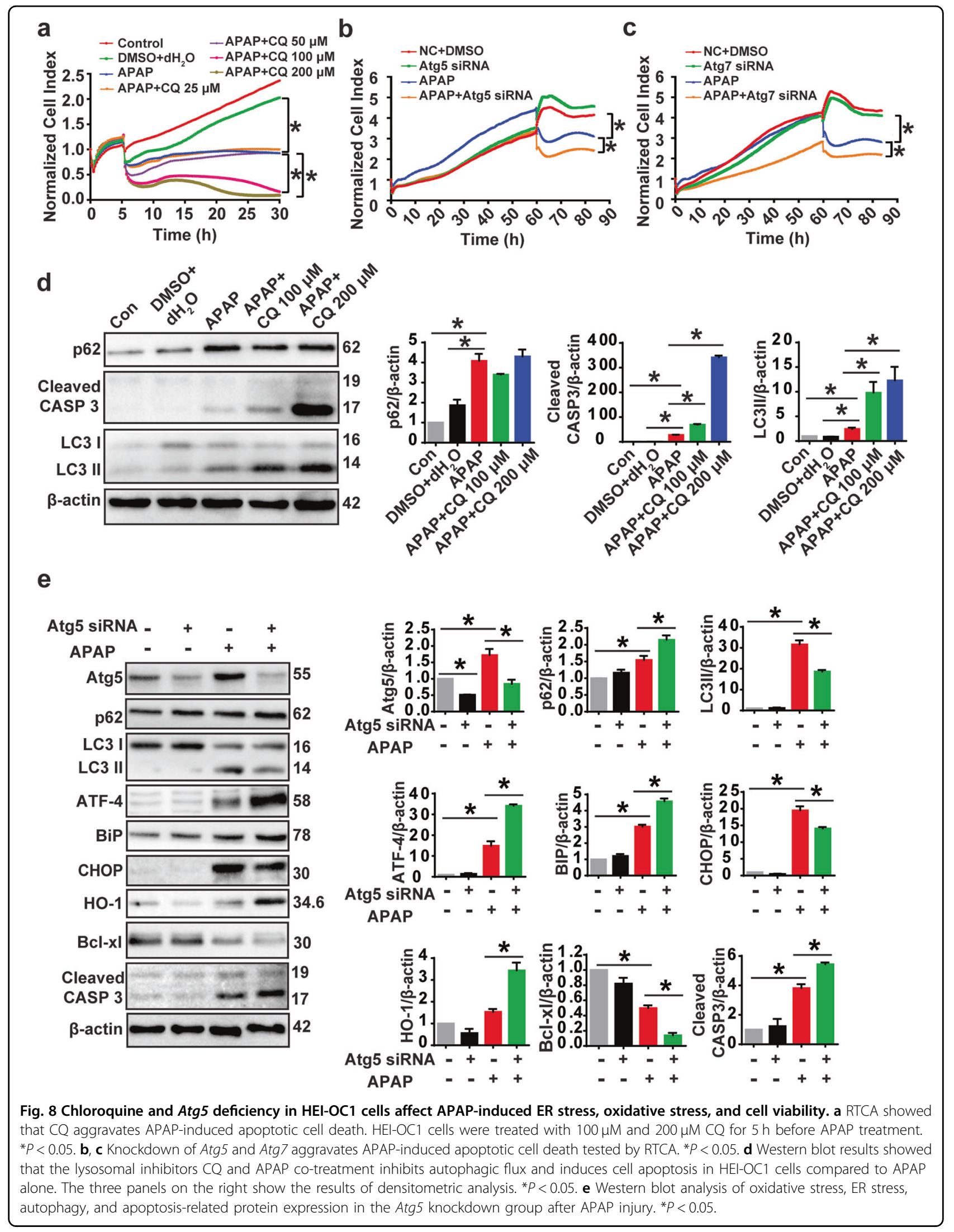


shown by the reduction of LC3-II and p62 protein levels. Autophagy impairment with lysosomal dysfunction is an important characteristic of oxidative stress-induced senescence $^{12}$. Emerging evidence suggests that ER stress can trigger autophagy ${ }^{68,69}$. However, our study showed that ER stress only have a weak, if any, contribution to APAPinduced autophagy impairment, because the effect of ER inhibitor on the alleviation of autophagy impairment is not obvious.

Previous studies have shown that there is a balance between oxidative stress and autophagy in sensory $\mathrm{HCs}^{13}$. In this study, we showed that knockdown of $\operatorname{Atg} 5$ or $\operatorname{Atg} 7$ decreased the expression of LC3-II and increased APAPinduced ROS levels and apoptotic cell death. As previously reported, there is a negative feedback mechanism between ER stress and autophagy ${ }^{70}$. Our results showed that, when $\operatorname{Atg} 5$ or $\operatorname{Atg} 7$ were knocked down in HEI-OC1 cells, APAP-induced ER stress levels and apoptotic cell death were increased. These results suggested that downregulation of autophagy leads to elevated ROS and ER stress levels, which further enhances apoptosis after APAP injury.

In summary, our study demonstrates that lysosomal impairment is an important factor contributing to APAPinduced cell death in HEI-OC1 cells and mice cochlear explants with impaired autophagy (Fig. S7). These findings highlight the potential of specific lysosomal activators in alleviating APAP-induced ototoxicity. Lysosome dysfunction may serve as a candidate target for therapeutic intervention, and future studies are warranted to design and test potential therapeutic treatments for preventing hearing loss caused by APAP.

\section{Acknowledgements}

This work was supported by grants from the National Natural Science Foundation of China (81873697, 81530030, 81600809, and 21605005); the Shandong Provincial Natural Science Foundation of China (ZR2018LH009); the Humanities and Social Sciences Foundation of the Ministry of Education of China (18YJC740128); the China Disabled Persons' Federation (CLS2019-02); the Taishan Scholar Foundation (ZR2019PH06) and the Clinical Medicine $+X$ academic degree program cultivation and construction project of Binzhou Medical University; Binzhou Medical University start-up fund to R.G.; and the National Institutes of Health of the United States (R01DC015111, R21DC005846, R01DK53307, and R01DK060596). We thank Dr. Federico Kalinec for providing the HEI-OC1 cell line.

\footnotetext{
Author details

'Hearing and Speech Rehabilitation Institute, College of Special Education, Binzhou Medical University, Yantai, China. ${ }^{2}$ Center for Hearing and Deafness, University at Buffalo, Buffalo, NY, USA. ${ }^{3}$ Department of Otolaryngology, Shenzhen Second People's Hospital, The First Affiliated Hospital of Shenzhen University, Shenzhen, China. ${ }^{4}$ Department of Genetics, School of Pharmacy, Binzhou Medical University, Yantai, China. ${ }^{5}$ Key Laboratory of Flexible Electronics \& Institute of Advanced Materials, Nanjing Tech University, Nanjing, China. ${ }^{6}$ Department of Otolaryngology-Head \& Neck Surgery, Second Affiliated Hospital, Xi'an Jiaotong University School of Medicine, Xi'an, China. ${ }^{7}$ School of Pharmacy, Guangdong Pharmaceutical University, Guangzhou, China. ${ }^{8}$ Department of Otolaryngology, Case Western Reserve University, Cleveland, OH, USA. ${ }^{9}$ Department of Genetics, Case Western Reserve University, Cleveland, $\mathrm{OH}$, USA
}

\section{Author contributions}

Investigation: B.L., T. Zheng, T. Zhao, H.Y., B.H., B.Y., and P.M.; resources: G.C.; writing-original draft: B.L. and T. Zhao; writing - review and editing: Q.Y.Z., T. Zhao, R.G., B.H.H., M.P., and N.Y.; preparation of figures: T. Zhao and B.L.; supervision: Q.Y.Z; funding acquisition: B.L., Q.Y.Z., Y.Y., J.H., T.C., and M.H. All authors read and approved the final manuscript.

\section{Conflict of interest}

The authors declare that they have no conflict of interest.

\section{Ethics statement}

The experimental protocol was initially approved by the Animal Care and Use Committee of the Binzhou Medical University, and the animal experiments were conducted under the National Institute Guide for the Care and Use of Laboratory Animals.

\section{Publisher's note}

Springer Nature remains neutral with regard to jurisdictional claims in published maps and institutional affiliations.

Supplementary Information accompanies this paper at (https://doi.org/ 10.1038/s41419-020-03328-6).

Received: 9 April 2020 Revised: 3 December 2020 Accepted: 7 December 2020

Published online: 04 January 2021

\section{References}

1. Gujal, J. S., Knight, T. R., Farhood, A., Bajt, M. L. \& Jaeschke, H. Mode of cell death after acetaminophen overdose in mice: apoptosis or oncotic necrosis? Toxicol. Sci. 67, 322-328 (2002).

2. Larson, A. M. Acetaminophen hepatotoxicity. Clin. Liver Dis. 11, 525-548 (2007).

3. Hinson, J. A., Roberts, D. W. \& James, L. P. Mechanisms of acetaminopheninduced liver necrosis. Handb. Exp. Pharmacol. 369-405 (2010).

4. McGill, M. R. \& Jaeschke, H. Metabolism and disposition of acetaminophen: recent advances in relation to hepatotoxicity and diagnosis. Pharmacol. Res. 30, 2174-2187 (2013).

5. Knight, T. R., Kurtz, A., Bajt, M. L., Hinson, J. A. \& Jaeschke, H. Vascular and hepatocellular peroxynitrite formation during acetaminophen toxicity: role of mitochondrial oxidant stress. Toxicol. Sci. 62, 212-220 (2001).

6. Xie, Y. et al. Mitochondrial protein adducts formation and mitochondrial dysfunction during $\mathrm{N}$-acetyl-m-aminophenol (AMAP)-induced hepatotoxicity in primary human hepatocytes. Toxicol. Appl. Pharmacol. 289, 213-222 (2015).

7. Ni, H. M. et al. Removal of acetaminophen protein adducts by autophagy protects against acetaminophen-induced liver injury in mice. J. Hepatol. 65, 354-362 (2016)

8. Mizushima, N., Levine, B., Cuervo, A. M. \& Klionsky, D. J. Autophagy fights disease through cellular self-digestion. Nature 451, 1069-1075 (2008).

9. Klionsky, D. J. \& Emr, S. D. Autophagy as a regulated pathway of cellular degradation. Science 290, 1717-1721 (2000).

10. Yadav, R. K. et al. TMBIM6 (transmembrane BAX inhibitor motif containing 6) enhances autophagy and reduces renal dysfunction in a cyclosporine Ainduced nephrotoxicity model. Autophagy 11, 1760-1774 (2015).

11. Levine, B. \& Yuan, J. Autophagy in cell death: an innocent convict? J. Clin. Investig. 115, 2679-2688 (2005).

12. Tai, H. et al. Autophagy impairment with lysosomal and mitochondrial dysfunction is an important characteristic of oxidative stress-induced senescence. Autophagy 13, 99-113 (2017).

13. He, Z. et al. Autophagy protects auditory hair cells against neomycin-induced damage. Autophagy 13, 1884-1904 (2017).

14. Kim, Y. J. et al. Autophagic flux, a possible mechanism for delayed gentamicininduced ototoxicity. Sci. Rep. 7, 41356 (2017).

15. Liu, Y. et al. Critical role of spectrin in hearing development and deafness. Sci. Adv. 5, eaav7803 (2019).

16. He, Z. H. et al. The nuclear transcription factor FoxG1 affects the sensitivity of mimetic aging hair cells to inflammation by regulating autophagy pathways. Redox Biol. 28, 101364 (2020). 
17. Gao, S. et al. Blebbistatin inhibits neomycin-induced apoptosis in hair cell-like HEl-OC-1 cells and in cochlear hair cells. Front. Cell. Neurosci. 13, 590 (2019).

18. Zhang, Y. et al. Pre-treatment with fasudil prevents neomycin-induced hair cell damage by reducing the accumulation of reactive oxygen species. Front. Mol. Neurosci. 12, 264 (2019).

19. Liu, W. et al. Wnt signaling activates TP53-induced glycolysis and apoptosis regulator and protects against cisplatin-induced spiral ganglion neuron damage in the mouse cochlea. Antioxid. Redox Signal. 30, 1389-1410 (2019).

20. Li, A. et al. Novel compounds protect auditory hair cells against gentamycininduced apoptosis by maintaining the expression level of H3K4me2. Drug Deliv. 25, 1033-1043 (2018).

21. Fu, X. L. et al. Tuberous sclerosis complex-mediated mTORC1 overactivation promotes age-related hearing loss. J. Clin. Investig. 128, 4938-4955 (2018).

22. $\mathrm{Li}, \mathrm{H}$. et al. Meclofenamic acid reduces reactive oxygen species accumulation and apoptosis, inhibits excessive autophagy, and protects hair cell-like HEIOC1 cells from cisplatin-induced damage. Front. Cell. Neurosci. 12, 139 (2018).

23. He, Z. H. et al. The role of FOXG1 in the postnatal development and survival of mouse cochlear hair cells. Neuropharmacology 144, 43-57 (2019).

24. Curhan, S. G., Eavey, R., Shargorodsky, J. \& Curhan, G. C. Analgesic use and the risk of hearing loss in men. Am. J. Med. 123, 231-237 (2010).

25. Curhan, S. G., Shargorodsky, J., Eavey, R. \& Curhan, G. C. Analgesic use and the risk of hearing loss in women. Am. J. Epidemiol. 176, 544-554 (2012).

26. Blakley, B. W. \& Schilling, H. Deafness associated with acetaminophen and codeine abuse. J. Otolaryngol. Head Neck Surg. 37, 507-509 (2008)

27. Oh, A. K., Ishiyama, A. \& Baloh, R. W. Deafness associated with abuse of hydrocodone/acetaminophen. Neurology 54, 2345 (2000).

28. Yorgason, J. G., Kalinec, G. M., Luxford, W. M., Warren, F. M. \& Kalinec, F. Acetaminophen ototoxicity after acetaminophen/hydrocodone abuse: evidence from two parallel in vitro mouse models. Otolaryngol. Head Neck Surg. 142, 814-819 (2010).

29. Kalinec, G. M. et al. Acetaminophen and NAPQI are toxic to auditory cells via oxidative and endoplasmic reticulum stress-dependent pathways. Hear Res. 313, 26-37 (2014).

30. Kalinec, G., Thein, P., Park, C. \& Kalinec, F. HEI-OC1 cells as a model for investigating drug cytotoxicity. Hear Res. 335, 105-117 (2016).

31. Sandilands, E. et al. Autophagic targeting of Src promotes cancer cell survival following reduced FAK signalling. Nat. Cell Biol. 14, 51-60 (2011).

32. Landegger, L. D., Dilwali, S. \& Stankovic, K. M. Neonatal murine cochlear explant technique as an in vitro screening tool in hearing research. J. Vis. Exp. https://doi.org/10.3791/55704 (2017).

33. Chen, F. Q., Schacht, J. \& Sha, S. H. Aminoglycoside-induced histone deacetylation and hair cell death in the mouse cochlea. J. Neurochem. 108, 1226-1236 (2009).

34. Dobretsov, M., Petkau, G., Hayar, A. \& Petkau, E. Clock scan protocol for image analysis: ImageJ plugins. J. Vis. Exp. https://doi.org/10.3791/55819 (2017).

35. Xu, M. et al. A phenotypic compound screening assay for lysosomal storage diseases. J. Biomol. Screen. 19, 168-175 (2014).

36. Valapala, M. et al. Impaired endolysosomal function disrupts Notch signalling in optic nerve astrocytes. Nat. Commun. 4, 1629 (2013).

37. Boya, P. \& Kroemer, G. Lysosomal membrane permeabilization in cell death. Oncogene 27, 6434-6451 (2008).

38. Ni, H. M. et al. Dissecting the dynamic turnover of GFP-LC3 in the autolysosome. Autophagy 7, 188-204 (2011).

39. Sergent, J. A., Paget, V. \& Chevillard, S. Toxicity and genotoxicity of nano-SiO2 on human epithelial intestinal HT-29 cell line. Ann. Occup. Hyg. 56, 622-630 (2012).

40. Kimura, S., Noda, T. \& Yoshimori, T. Dissection of the autophagosome maturation process by a novel reporter protein, tandem fluorescent-tagged LC3. Autophagy 3, 452-460 (2007).

41. Hyttinen, J. M. T., Niittykoski, M., Salminen, A. \& Kaarniranta, K. Maturation of autophagosomes and endosomes: a key role for Rab7. Biochim. Biophys. Acta 1833, 503-510 (2013).

42. Klionsky, D. J. et al. Guidelines for the use and interpretation of assays for monitoring autophagy (3rd edition). Autophagy 12, 1-222 (2016).

43. Dehay, B. et al. Lysosomal impairment in Parkinson's disease. Mov. Disord. 28, 725-732 (2013).
44. Settembre, C. et al. TFEB links autophagy to lysosomal biogenesis. Science $\mathbf{3 3 2}$ 1429-1433 (2011)

45. Filomeni, G., De Zio, D. \& Cecconi, F. Oxidative stress and autophagy: the clash between damage and metabolic needs. Cell Death Differ. 22, 377-388 (2015).

46. Gao, Q. Oxidative stress and autophagy. Adv. Exp. Med. Biol. 1206, 179-198 (2019).

47. Lee, W. S., Yoo, W. H. \& Chae, H. J. ER stress and autophagy. Curr. Mol. Med. 15, 735-745 (2015).

48. Song, S., Tan, J., Miao, Y., Li, M. \& Zhang, Q. Crosstalk of autophagy and apoptosis: Involvement of the dual role of autophagy under ER stress. J. Cell. Physiol. 232, 2977-2984 (2017).

49. Qi, J. Y. et al. A cytoskeleton structure revealed by super-resolution fluorescence imaging in inner ear hair cells. Cell Discov. 5, 12 (2019).

50. Qi, J. et al. Espin distribution as revealed by super-resolution microscopy of stereocilia. Am. J. Transl. Res. 12, 130-141 (2020).

51. Tan, F. et al. AAV-ie enables safe and efficient gene transfer to inner ear cells. Nat. Commun. 10, 3733 (2019).

52. Cheng, C. et al. Age-related transcriptome changes in Sox2+supporting cells in the mouse cochlea. Stem Cell Res. Ther. 10, 365 (2019).

53. Zhang, S. et al. Frizzled-9+ supporting cells are progenitors for the generation of hair cells in the postnatal mouse cochlea. Front. Mol. Neurosci. 12, 184 (2019).

54. Lu, X. L. et al. Bmi1 regulates the proliferation of cochlear supporting cells via the canonical Wnt signaling pathway. Mol. Neurobiol. 54, 1326-1339 (2017).

55. Wang, T. et al. Lgr5+cells regenerate hair cells via proliferation and direct transdifferentiation in damaged neonatal mouse utricle. Nat. Commun. 6, 6613 (2015).

56. Cox, B. C. et al. Spontaneous hair cell regeneration in the neonatal mouse cochlea in vivo. Development 141, 816-829 (2014).

57. Liu, L. et al. Wnt activation protects against neomycin-induced hair cell damage in the mouse cochlea. Cell Death Dis. 7, E2136 (2016).

58. Zhu, C. W. et al. Loss of ARHGEF6 causes hair cell stereocilia deficits and hearing loss in mice. Front. Mol. Neurosci. 11, 362 (2018).

59. Wang, Y. F. et al. Loss of CIB2 causes profound hearing loss and abolishes mechanoelectrical transduction in mice. Front. Mol. Neurosci. 10, 401 (2017).

60. Zhang, S. S. et al. Characterization of Lgr5+ progenitor cell transcriptomes after neomycin injury in the neonatal mouse cochlea. Front. Mol. Neurosci. 10, 213 (2017).

61. $Y u, X$. Y. et al. c-Myb knockdown increases the neomycin-induced damage to hair-cell-like HEl-OC1 cells in vitro. Sci. Rep. 7, 41094 (2017).

62. Guan, M. et al. Inhibition of ARC decreases the survival of HEl-OC-1 cells after neomycin damage in vitro. Oncotarget 7, 66647-66659 (2016).

63. He, Z. H. et al. Reduced TRMU expression increases the sensitivity of hair-celllike HEI-OC-1 cells to neomycin damage in vitro. Sci. Rep. 6, 29621 (2016).

64. Sun, S. et al. In vivo overexpression of X-linked inhibitor of apoptosis protein protects against neomycin-induced hair cell loss in the apical turn of the cochlea during the ototoxic-sensitive period. Front. Cell. Neurosci. 8 , 248 (2014)

65. Yan, W. Q. et al. A three-dimensional culture system with matrigel promotes purified spiral ganglion neuron survival and function in vitro. Mol. Neurobiol. 55, 2070-2084 (2018)

66. George, A. A. et al. Synaptojanin 1 is required for endolysosomal trafficking of synaptic proteins in cone photoreceptor inner segments. PLoS ONE 9, e84394 (2014).

67. Wang, Y. Q., Nartiss, Y., Steipe, B., McQuibban, G. A. \& Kim, P. K. ROS-induced mitochondrial depolarization initiates PARK2/PARKIN-dependent mitochondrial degradation by autophagy. Autophagy 8, 1462-1476 (2012).

68. Nie, T. J. et al. Regulation of ER stress-induced autophagy by GSK3 beta-TIP60ULK1 pathway. Cell Death Dis. 7, E2563 (2016).

69. Song, S. L., Tan, J., Miao, Y. Y. \& Zhang, Q. Crosstalk of ER stress-mediated autophagy and ER-phagy: involvement of UPR and the core autophagy machinery. J. Cell. Physiol. 233, 3867-3874 (2018).

70. Senft, D. \& Ronai, Z. A. UPR, autophagy, and mitochondria crosstalk underlies the ER stress response. Trends Biochem. Sci. 40, 141-148 (2015). 